\title{
Generalized gauge transformations: Pure Yang-Mills case
}

\author{
R. Gastmans* \\ Institute for Theoretical Physics, University of Leuven, B-3001 Leuven, Belgium \\ Tai Tsun $\mathrm{Wu}$ \\ Gordon McKay Laboratory, Harvard University, Cambridge, Massachusetts 02138 \\ and Theory Division, CERN, CH-1211 Geneva 23, Switzerland
}

(Received 20 August 1997; published 23 December 1997)

\begin{abstract}
Gauge transformations with Dirac point splitting are systematically discussed for the case of a pure YangMills theory. These generalized gauge transformations are based on two ingredients: a fixed four-vector, which defines the point splitting, and a weight function, which gives an average over the amount of point splitting and which provides a cutoff in momentum space in the direction of the point splitting four-vector. From the requirement that the group property must be satisfied, it is found, starting from a simple ansatz, that an infinitesimal generalized gauge transformation takes the form of an infinite series in the coupling constant. Using induction on the order of the coupling constant, it is shown that all higher-order terms indeed exist and that they can be expressed in terms of the lower-order formulas. That there are such generalized gauge transformations suggests the possibility of a Yang-Mills field theory with mitigated divergences. [S0556-2821(97)03524-8]
\end{abstract}

PACS number(s): 11.15.- $\mathrm{q}$

\section{INTRODUCTION}

Point splitting, as a remedy for avoiding divergences in relativistic quantum field theory, has a long history. Over 60 years ago, Dirac [1] pointed out that by introducing a fixed four-vector $\epsilon_{\mu}$ and by replacing products of field operators $A(x) B(x)$ by $A(x-\epsilon) B(x+\epsilon)$, the divergence problem could be circumvented. This suggests that all calculations should be performed for finite values of $\epsilon_{\mu}$, and that, only at the end, one should take the limit $\epsilon_{\mu} \rightarrow 0$. Various attempts have been presented $[2,3]$, in which one tries to make use of this attractive idea.

It is the purpose of this paper to provide the first systematic discussion of gauge transformations for the case of the pure Yang-Mills theory with point splitting. Gauge transformations incorporating the idea of point splitting will be referred to as generalized gauge transformations, and will be given explicitly.

The starting point of the present investigation consists of (1) in the Dirac point splitting, a direction, that of $\epsilon_{\mu}$, is picked out as the direction of the splitting, and (2) in order to mitigate divergences, a momentum cutoff needs to be introduced. With these observations, it is natural to choose the momentum cutoff in the direction of $\epsilon_{\mu}$. In other words, we choose the momentum cutoff at

$$
|k \cdot \epsilon|<1 \text {. }
$$

In the limit $\epsilon_{\mu} \rightarrow 0$, this momentum cutoff is taken to infinity, i.e., there is no cutoff.

In Yang-Mills theory, the infinitesimal gauge transformation is

*Electronic address: raymond.gastmans@fys.kuleuven.ac.be

$$
\delta_{\Lambda} \mathbf{A}_{\mu}(x)=-\partial_{\mu} \mathbf{\Lambda}(x)-\lg \left[\mathbf{\Lambda}(x), \mathbf{A}_{\mu}(x)\right] .
$$

In momentum space, this takes the form

$$
\begin{aligned}
\delta_{\Lambda} \mathbf{A}_{\mu}(k)= & \iota k_{\mu} \Lambda(k)-\imath g \int d^{4} k_{1} \int d^{4} k_{2} \delta^{(4)}\left(k-k_{1}-k_{2}\right) \\
& \times\left[\mathbf{\Lambda}\left(k_{1}\right), \mathbf{A}_{\mu}\left(k_{2}\right)\right] .
\end{aligned}
$$

When the momentum cutoff (1) is introduced, Eq. (3) is reinterpreted as

$$
\begin{aligned}
\delta_{\Lambda} \mathbf{A}_{\mu}(k)= & \iota k_{\mu} \mathbf{\Lambda}(k)-\imath g \int_{K} d^{4} k_{1} \int_{K} d^{4} k_{2} \delta^{(4)}\left(k-k_{1}-k_{2}\right) \\
& \times\left[\boldsymbol{\Lambda}\left(k_{1}\right), \mathbf{A}_{\mu}\left(k_{2}\right)\right]
\end{aligned}
$$

when $k$ is in $K$, the region $K$ being defined by Eq. (1). In other words, $k, k_{1}$, and $k_{2}$ are all required to satisfy the inequality (1).

In order for Eq. (4) to define a gauge transformation, it must satisfy

$$
\left[\delta_{\Lambda_{1}}, \delta_{\Lambda_{2}}\right] \mathbf{A}_{\mu}(k)=\delta_{L} \mathbf{A}_{\mu}(k)
$$

exactly, where $\delta_{L}$ is a gauge transformation that, of course, depends on $\boldsymbol{\Lambda}_{1}$ and $\boldsymbol{\Lambda}_{2}$. Unlike Eq. (3), Eq. (4) does not satisfy Eq. (5) exactly, and, therefore, higher-order terms in $g$ must be added to it. For the same reason, $\mathbf{L}$ must also take on the form of an infinite series. The main part of this paper is precisely devoted to the derivation of explicit formulas for $\delta_{\Lambda} \mathbf{A}_{\mu}(k)$ and $\mathbf{L}$ to all orders in $g$. Because we do not make use of any specific properties of the structure constants, the results we shall obtain are valid for an arbitrary Yang-Mills symmetry.

This paper is organized as follows. In Sec. II, we introduce our notation. In Sec. III, we work out explicitly the first 
and second order in the generalized gauge transformation, and, in Sec. IV, we work out the general formula for arbitrary order. We present explicit formulas which express the gauge transformation and the combined gauge parameter to a given order in terms of lower-order expressions. Our procedure to arbitrary order requires the use of two identities which are proven in the Appendixes. In Sec. V, the relation to some previous papers are discussed briefly. Finally, Sec. VI presents the conclusions.

\section{NOTATION}

\section{A. Lie algebra}

A Lie algebra is characterized by a set of generators $T_{a}$ which satisfy the commutation relations

$$
\left[T_{a}, T_{b}\right]={ }_{l} f_{a b}^{c} T_{c},
$$

where the quantities $f_{a b}^{c}$ are the structure constants of the Lie algebra. In the standard case, without point splitting, the transformation of the gauge fields is given by

$$
\delta_{\Lambda} A_{\mu}^{a}(x)=-\partial_{\mu} \Lambda^{a}(x)+g f_{b c}^{a} \Lambda^{b}(x) A_{\mu}^{c}(x),
$$

where $\Lambda^{a}(x)$ are the infinitesimal gauge parameters.

Introducing the group elements $\mathbf{A}_{\mu}(x) \equiv T_{a} A_{\mu}^{a}(x)$ and $\Lambda(x) \equiv T_{a} \Lambda^{a}(x)$, Eq. (7) is the same as Eq. (2). The only property of the Lie algebra that we shall use is the Jacobi identity

$$
[\mathbf{A},[\mathbf{B}, \mathbf{C}]]+[\mathbf{B},[\mathbf{C}, \mathbf{A}]]+[\mathbf{C},[\mathbf{A}, \mathbf{B}]]=0 .
$$

Throughout the rest of the paper, we shall only consider group elements, and, to alleviate the formulas, we shall drop the boldface notation for these quantities. This means that $A_{\mu}$ and $\Lambda$ now stand for the group elements themselves.

\section{B. Momentum space}

In Eq. (4), the Fourier transforms are defined by

$$
\begin{gathered}
A_{\mu}(k)=\frac{1}{(2 \pi)^{2}} \int d^{4} x e^{\imath k \cdot x} A_{\mu}(x), \\
\Lambda(k)=\frac{1}{(2 \pi)^{2}} \int d^{4} x e^{\imath k \cdot x} \Lambda(x) .
\end{gathered}
$$

It is convenient to introduce the function $R$ defined by

$$
R(k \cdot \epsilon)= \begin{cases}1 & \text { for }|k \cdot \epsilon|<1, \\ 0 & \text { for }|k \cdot \epsilon| \geqslant 1 .\end{cases}
$$

Then Eq. (4) can be rewritten as

$$
\begin{aligned}
\delta_{\Lambda} A_{\mu}(k)= & R(k \cdot \epsilon){ }_{l} k_{\mu} \Lambda(k)-R(k \cdot \epsilon){ }_{l} g \int d^{4} k_{1} \int d^{4} k_{2} \\
& \times \delta^{(4)}\left(k-k_{1}-k_{2}\right) R\left(k_{1} \cdot \epsilon\right) R\left(k_{2} \cdot \epsilon\right) \\
& \times\left[\Lambda\left(k_{1}\right), A_{\mu}\left(k_{2}\right)\right]+\mathcal{O}\left(g^{2}\right),
\end{aligned}
$$

provided that the condition is used where

$$
\delta_{\Lambda} A_{\mu}(k)=0 \text { for }|k \cdot \epsilon| \geqslant 1 .
$$

Since we have to deal with arbitrary powers of $g$, it is essential to simplify the notation. Using Eq. (11) as the example, the first step is to drop all $\cdot \boldsymbol{\epsilon}$ since there is no confusion:

$$
\begin{aligned}
\delta_{\Lambda} A_{\mu}(k)= & R(k)_{l} k_{\mu} \Lambda(k)-R(k) \lg \int d^{4} k_{1} \int d^{4} k_{2} \\
& \times \delta^{(4)}\left(k-k_{1}-k_{2}\right) R\left(k_{1}\right) R\left(k_{2}\right)\left[\Lambda\left(k_{1}\right), A_{\mu}\left(k_{2}\right)\right] \\
& +\mathcal{O}\left(g^{2}\right) .
\end{aligned}
$$

Thus the arguments of $A_{\mu}$ and $\Lambda$ are the four-vectors $k, k_{1}$, or $k_{2}$, while the arguments of the $R$ functions are the scalars $k, k_{1}$, or $k_{2}$, meaning $k \cdot \epsilon, k_{1} \cdot \epsilon$, or $k_{2} \cdot \epsilon$.

To simplify the notation further, we drop the integral signs and the $\delta$ function. Moreover, the arguments $k_{i}$ will be denoted by $i$, except for the argument $k$ which remains unchanged. With these conventions, Eq. (13) becomes

$$
\begin{aligned}
\delta_{\Lambda} A_{\mu}(k)= & R(k) l k_{\mu} \Lambda(k)-R(k) \lg R(1) R(2)\left[\Lambda(1), A_{\mu}(2)\right] \\
& +\mathcal{O}\left(g^{2}\right) .
\end{aligned}
$$

We also find it convenient to introduce the function $S(k)$ defined by

$$
S(k)=1-R(k) .
$$

Obviously, for all values of $k$,

$$
R^{2}(k)=R(k), \quad S^{2}(k)=S(k), \quad R(k) S(k)=0 .
$$

The arguments of the $S$ functions are treated on the same footing as the $R$ functions.

\section{FIRST AND SECOND ORDER}

In Sec. II, it has been shown that the ideas of point splitting in position space translate into the appearance of the function $R(k)$ in momentum space, which provides a cutoff for large values of the momentum $k$. The purpose of this paper is to show the way to satisfy the group property

$$
\left[\delta_{\Lambda_{1}}, \delta_{\Lambda_{2}}\right] A_{\mu}(k)=\delta_{L} A_{\mu}(k),
$$

where $L(k)$ is the combined gauge parameter. In Eq. (17), the gauge parameters $\Lambda_{i}, i=1,2$ are taken to be infinitesimal.

Anticipating on the result, we expand the gauge transformation and the combined gauge parameter in powers of the coupling constant $g$ :

$$
\delta_{\Lambda} A_{\mu}(k)=\sum_{n=0}^{\infty} g^{n} \delta_{\Lambda}^{(n)} A_{\mu}(k), \quad L(k)=\sum_{n=1}^{\infty} g^{n} L^{(n)}(k) .
$$

It follows from Eqs. (14) and (18) that

$$
\begin{gathered}
\delta_{\Lambda}^{(0)} A_{\mu}(k)={ }_{l} R(k) k_{\mu} \Lambda(k), \\
\delta_{\Lambda}^{(1)} A_{\mu}(k)=-{ }_{l} R(1+2) R(1) R(2)\left[\Lambda(1), A_{\mu}(2)\right] .
\end{gathered}
$$


To first order in $g$, the group property requires

$$
\left[\delta_{\Lambda_{2}}^{(0)} \delta_{\Lambda_{1}}^{(1)}-\delta_{\Lambda_{1}}^{(0)} \delta_{\Lambda_{2}}^{(1)}\right] A_{\mu}(k)=\imath k_{\mu} L^{(1)}(k)
$$

The left-hand side (LHS) of this equation reads

$$
\begin{aligned}
R(1+2) R(1) R(2)\left\{\left[\Lambda_{1}(1), k_{2 \mu} \Lambda_{2}(2)\right]\right. \\
\left.\quad-\left[\Lambda_{2}(1), k_{2 \mu} \Lambda_{1}(2)\right]\right\} \\
=k_{\mu} R(1+2) R(1) R(2)\left[\Lambda_{1}(1), \Lambda_{2}(2)\right],
\end{aligned}
$$

because of the implicit integrations and $\delta$ function in this formula. It follows that the condition (20) is satisfied provided we take

$$
L^{(1)}(k)=-{ }_{l} R(1+2) R(1) R(2)\left[\Lambda_{1}(1), \Lambda_{2}(2)\right] .
$$

Note that in the limit $\epsilon \rightarrow 0$, both the zeroth order and the first order gauge transformation in Eqs. (19), as well as the combined gauge parameter $L^{(1)}(k)$ in Eq. (22), reduce to the standard expressions without point splitting, because, in that limit, all the arguments of the $R$ functions tend to zero, and $R(0)=1$.

To order $g^{2}$, the formulas become somewhat more complicated. We therefore introduce some more notation in order to limit the size of the formulas and to improve their readability. The group property (17) always refers to commutators of two successive gauge transformations. To avoid writing down this type of commutators, we propose to write only the first term of the commutator, the second term with the $\Lambda_{1} \leftrightarrow \Lambda_{2}$ interchange being always understood. With this convention, the expression for $L^{(1)}(k)$, e.g., becomes

$$
L^{(1)}(k)=-\frac{l}{2} R(1+2) R(1) R(2)\left[\Lambda_{1}(1), \Lambda_{2}(2)\right] .
$$

To order $g^{2}$, the group property (17) now reads

$$
\left[\delta_{\Lambda_{2}}^{(1)} \delta_{\Lambda_{1}}^{(1)}+\delta_{\Lambda_{2}}^{(0)} \delta_{\Lambda_{1}}^{(2)}\right] A_{\mu}(k)={ }_{l} k_{\mu} L^{(2)}(k)-{ }_{l} R(1+2) R(1) R(2)\left[L^{(1)}(1), A_{\mu}(2)\right] .
$$

We thus have to find $L^{(2)}(k)$ and $\delta_{\Lambda}^{(2)} A_{\mu}(k)$ such that Eq. (24) is satisfied. Let us rewrite Eq. (24) in such a way that the terms which are known from the order $g$ calculation appear on the LHS:

$$
\delta_{\Lambda_{2}}^{(1)} \delta_{\Lambda_{1}}^{(1)} A_{\mu}(k)+{ }_{\imath} R(1+2) R(1) R(2)\left[L^{(1)}(1), A_{\mu}(2)\right]={ }_{l} k_{\mu} L^{(2)}(k)-\delta_{\Lambda_{2}}^{(0)} \delta_{\Lambda_{1}}^{(2)} A_{\mu}(k) .
$$

In Eq. (25), the LHS is explicitly given by

$$
\begin{aligned}
& -R(1+2+3) R(1) R(2+3) R(2) R(3)\left\{\left[\Lambda_{1}(1),\left[\Lambda_{2}(2), A_{\mu}(3)\right]\right]+\frac{1}{2}\left[A_{\mu}(1),\left[\Lambda_{1}(2), \Lambda_{2}(3)\right]\right]\right\} \\
& \quad=R(1+2+3) R(1) S(2+3) R(2) R(3)\left\{\left[\Lambda_{1}(1),\left[\Lambda_{2}(2), A_{\mu}(3)\right]\right]+\frac{1}{2}\left[A_{\mu}(1),\left[\Lambda_{1}(2), \Lambda_{2}(3)\right]\right]\right\} .
\end{aligned}
$$

Note that in rewriting the expression (26) we used the fact that, without a factor $R(2+3)$, the expression would have vanished by virtue of the Jacobi identity. Hence, we could replace $R(2+3)$ by $-S(2+3)$.

A simple calculation shows that Eq. (24) is satisfied if we take

$$
\begin{aligned}
\delta_{\Lambda}^{(2)} A_{\mu}(k)= & -\imath R(1+2+3) R(1) S(2+3) R(2) R(3) \\
& \times\left\{\frac{1}{\epsilon \cdot k_{1}}\left[\epsilon \cdot A(1),\left[\Lambda(2), A_{\mu}(3)\right]\right]\right. \\
& +\frac{k_{1 \mu}}{\epsilon \cdot k_{1} \epsilon \cdot\left(k_{2}+k_{3}\right)}[\epsilon \cdot A(1),[\Lambda(2), \epsilon \cdot A(3)]] \\
& \left.-\frac{1}{\epsilon \cdot\left(k_{2}+k_{3}\right)}\left[A_{\mu}(1),[\Lambda(2), \epsilon \cdot A(3)]\right]\right\},
\end{aligned}
$$

and

$$
\begin{aligned}
L^{(2)}(k)= & -\imath R(1+2+3) R(1) S(2+3) R(2) R(3) \frac{1}{2 \epsilon \cdot k_{1}} \\
& \times\left[\epsilon \cdot A(1),\left[\Lambda_{1}(2), \Lambda_{2}(3)\right]\right] .
\end{aligned}
$$

A few comments should be made about the formulas (27) and (28). In the limit $\epsilon \rightarrow 0$, the expressions for both $\delta_{\Lambda}^{(2)} A_{\mu}(k)$ and $L^{(2)}(k)$ vanish because the argument of the $S$ function tends to zero and $S(0)=0$. This has to be so, because, in the standard case without point splitting, there is no second-order term neither in the gauge transformation nor in the combined gauge parameter.

Also, we could have chosen different expressions for $\delta_{\Lambda}^{(2)} A_{\mu}(k)$ and $L^{(2)}(k)$ and still satisfy the group property. We imposed, however, the requirement that the expressions 
be free of singularities. One can verify that the potential singularity for $\epsilon \cdot k_{1}=0$ is not present, because the numerator has a factor $R(1+2+3) S(2+3)$. In the limit $\epsilon \cdot k_{1} \rightarrow 0$, this factor tends to $R(2+3) S(2+3)=0$. Similarly, for $\epsilon \cdot\left(k_{2}+k_{3}\right) \rightarrow 0$, the factor $S(2+3)$ tends to $S(0)=0$. Still, the requirement of absence of singularities does not uniquely determine the expressions for $\delta_{\Lambda}^{(2)} A_{\mu}(k)$ and $L^{(2)}(k)$. In our experience, however, the choices in Eqs. (27) and (28) lead to the simplest results in higher orders. For that reason, we do not examine the other possibilities here.

Again, we introduce more simplifications in the notation. All denominators which we encounter, apart from numerical factors, are of the form $\epsilon \cdot k_{i}$ (or sums of such expressions). Hence, without creating confusion, we can simply denote them by $k_{i}$. Also, the expressions $\epsilon \cdot A\left(k_{i}\right)$ will be denoted in what follows by $A(i)$. Also, it will appear that the factors $A(i)$ always have a factor $R(i)$ associated with them. We therefore can drop the factors $R(i)$ in the simplified notation. Finally, we want to develop some recursive procedure to prove the existence of the generalized gauge transformation to all orders. For that reason, it is advantageous to express higher-order formulas for $\delta_{\Lambda}^{(n)} A_{\mu}(k)$ and $L^{(n)}(k)$ in terms of lower-order formulas. It turns out that the expressions $\delta_{\Lambda}^{(\ell)} A_{\mu}\left(k_{i}\right)$ and $L^{(\ell)}\left(k_{i}\right)$ have a factor $R(i), S(i)$, or unity associated with them. Most of the time, it is the factor $S(i)$ which is present. Whenever this is the case, we shall also drop these factors $S(i)$. When the $R(i)$ factor is present, we shall explicitly write it, and when the unit factor appears, we shall write $\overline{\delta_{\Lambda}^{(\ell)} A_{\mu}(i)}$ or $\overline{L^{(\ell)}(i)}$.

In the simplified notation, the formulas (27) and (28) now read

$$
\begin{aligned}
\delta_{\Lambda}^{(2)} A_{\mu}(k)= & R(1+2)\left\{\frac{1}{k_{1}}\left[A(1), \widetilde{\delta}_{\Lambda}^{(1)} A_{\mu}(2)\right]\right. \\
& +\frac{k_{1 \mu}}{k_{1} k_{2}}\left[A(1), \widetilde{\delta}_{\Lambda}^{(1)} A(2)\right] \\
& \left.-\frac{1}{k_{2}}\left[A_{\mu}(1), \widetilde{\delta}_{\Lambda}^{(1)} A(2)\right]\right\},
\end{aligned}
$$

and

$$
L^{(2)}(k)=\frac{R(1+2)}{k_{1}}\left[A(1), \widetilde{L}^{(1)}(2)\right],
$$

where, in Eqs. (29) and (30),

$$
\begin{aligned}
& \widetilde{\delta}_{\Lambda}^{(1)} A_{\mu}(k)=-\imath\left[\Lambda(1), A_{\mu}(2)\right], \\
& \widetilde{L}^{(1)}(k)=-\frac{l}{2}\left[\Lambda_{1}(1), \Lambda_{2}(2)\right] .
\end{aligned}
$$

\section{ARBITRARY ORDER}

We shall prove that the group property can be satisfied to arbitrary order. Let

$$
\delta_{\Lambda}^{(n)} A_{\mu}(k)=R(k) \widetilde{\delta}_{\Lambda}^{(n)} A_{\mu}(k), \quad L^{(n)}(k)=R(k) \widetilde{L}^{(n)}(k),
$$

with

$$
\begin{gathered}
\widetilde{\delta}_{\Lambda}^{(0)} A_{\mu}(k)={ }_{l} k_{\mu} \Lambda(k), \quad \widetilde{\delta}^{(1)} A_{\mu}(k)=-\imath\left[\Lambda(1), A_{\mu}(2)\right], \\
\widetilde{L}^{(1)}(k)=-\frac{l}{2}\left[\Lambda_{1}(1), \Lambda_{2}(2)\right],
\end{gathered}
$$

and, for $n \geqslant 2$,

$$
\begin{aligned}
\widetilde{\delta}^{(n)} A_{\mu}(k)= & -\sum_{a=1}^{n-1}(-1)^{a}\left\{\frac{1}{\left(k_{1}+\cdots+k_{a}\right) \cdots k_{a}}\left[A(1),\left[\ldots,\left[A(a), \widetilde{\delta}_{\Lambda}^{(n-a)} A_{\mu}(a+1)\right] \ldots\right]\right]\right. \\
& +\frac{\left(k_{1}+\cdots+k_{a}\right)_{\mu}}{\left(k_{1}+\cdots+k_{a}\right) \cdots k_{a} k_{a+1}}\left[A(1),\left[\ldots,\left[A(a), \widetilde{\delta}_{\Lambda}^{(n-a)} A(a+1)\right] \ldots\right]\right] \\
& \left.-\frac{1}{\left(k_{2}+\cdots+k_{a}\right) \cdots k_{a} k_{a+1}}\left[A_{\mu}(1),\left[A(2),\left[\ldots,\left[A(a), \widetilde{\delta}_{\Lambda}^{(n-a)} A(a+1)\right] \ldots\right]\right]\right]\right\},
\end{aligned}
$$

and

$$
\begin{aligned}
\widetilde{L}^{(n)}(k)= & -\sum_{a=1}^{n-1} \frac{(-1)^{a}}{\left(k_{1}+\cdots+k_{a}\right) \cdots k_{a}}\left[A(1),\left[\ldots,\left[A(a), \widetilde{L}^{(n-a)}(a+1)\right] \ldots\right]\right] \\
& -i \sum_{a=0}^{n-3} \sum_{l=1}^{n-a-2} \frac{(-1)^{a}\left[A(1),\left[\ldots,\left[A(a),\left[\widetilde{\delta}_{\Lambda_{1}}^{(\ell)} A(a+1), \widetilde{\delta}_{\Lambda_{2}}^{(n-\ell-a-1)} A(a+2)\right]\right] \ldots\right]\right]}{2\left(k_{1}+\cdots+k_{a}\right) \cdots k_{a} k_{a+1} k_{a+2}} .
\end{aligned}
$$

In Eqs. (34), (35), and throughout the rest of the paper, we use the standard summation convention that

$$
\sum_{a}^{b}=0 \quad \text { for } \quad b=a-1
$$

and that 


$$
\frac{1}{\left(k_{a}+\cdots+k_{b}\right) \cdots k_{b}}=\frac{1}{k_{a} \cdots\left(k_{a}+\cdots+k_{b}\right)}=1 \quad \text { for } \quad b=a-1
$$

We also point out that, in Eqs. (34) and (35), the quantities $\widetilde{\delta}_{\Lambda}^{(m)} A(i)$ and $\widetilde{L}^{(m)}(i)$ have factors $S(i)$ implicitly associated with them, while all other quantities have implicit $R$ factors.

We want to prove the group property, which reads to order $n$ :

$$
\sum_{\ell=0}^{n-1}\left(\delta_{\Lambda_{2}}^{(\ell)} \delta_{\Lambda_{1}}^{(n-\ell)}-\delta_{L^{(n-\ell)}}^{(\varrho)} A_{\mu}(k)=0 .\right.
$$

To this end, we shall first prove the somewhat simpler relation which reads

$$
\sum_{\ell=0}^{n-1}\left(\delta_{\Lambda_{2}}^{(\ell)} \widetilde{\delta}_{\Lambda_{1}}^{(n-\ell)}-\widetilde{\delta}_{\widetilde{L}^{(n-\ell)}}^{(\varrho)} A_{\mu}(k)=0 .\right.
$$

We intend to prove that Eq. (39) is valid using the induction method, i.e., we shall assume that, for $a=1,2, \ldots, n-1$,

$$
\sum_{\ell=0}^{n-a-1}\left(\delta_{\Lambda_{2}}^{(\ell)} \widetilde{\delta}_{\Lambda_{1}}^{(n-a-\ell)}-\widetilde{\delta}_{\left.\widetilde{L}^{(n-a-}\right)}^{()}\right) A_{\mu}(k)=0
$$

From the discussion in Sec. II, we already know that Eq. (39) is satisfied for $n=1,2$.

For the proof, it is useful to note that the expressions for $\widetilde{\delta}_{\Lambda}^{(n)} A_{\mu}(k)$ simplify when $\mu$ is in the direction of $\epsilon$. For $n \geqslant 2$,

$$
\widetilde{\delta}_{\Lambda}^{(n)} A(k)=-\sum_{a=1}^{n-1} \frac{(-1)^{a}}{\left(k_{1}+\cdots+k_{a}\right) \cdots k_{a}}\left[A(1),\left[\ldots,\left[A(a), \widetilde{\delta}_{\Lambda}^{(n-a)} A(a+1)\right] \ldots\right]\right] .
$$

It is not difficult to obtain the explicit expressions for the various terms in Eq. (39), using the formulas (34) and (35). We simply list the results, which are somewhat lengthy:

$$
\begin{aligned}
& \delta_{\Lambda_{2}}^{(0)} \widetilde{\delta}_{\Lambda_{1}}^{(n)} A_{\mu}(k)={ }_{l}\left[\Lambda_{1}(1), R(2) \delta_{\Lambda_{2}}^{(n-1)} A_{\mu}(2)\right]-\sum_{a=1}^{n-1} \frac{(-1)^{a}}{\left(k_{1}+\cdots+k_{a}\right) \cdots k_{a}}\left[A(1),\left[\ldots,\left[A(a), \delta_{\Lambda_{1}}^{(0)} \widetilde{\delta}_{\Lambda_{2}}^{(n-a)} A_{\mu}(a+1)\right] \ldots\right]\right] \\
& -\sum_{a=1}^{n-1} \frac{(-1)^{a}\left(k_{1}+\cdots+k_{a}\right)_{\mu}}{\left(k_{1}+\cdots+k_{a}\right) \cdots k_{a} k_{a+1}}\left[A(1),\left[\ldots,\left[A(a), \delta_{\Lambda_{1}}^{(0)} \widetilde{\delta}_{\Lambda_{2}}^{(n-a)} A(a+1)\right] \ldots\right]\right] \\
& +\sum_{a=1}^{n-1} \frac{(-1)^{a}}{\left(k_{2}+\cdots+k_{a}\right) \cdots k_{a} k_{a+1}}\left[A_{\mu}(1),\left[\ldots,\left[A(a), \delta_{\Lambda_{1}}^{(0)} \widetilde{\delta}_{\Lambda_{2}}^{(n-a)} A(a+1)\right] \ldots\right]\right] \\
& +\sum_{a=2}^{n-1} \frac{(-1)^{a}}{\left(k_{1}+\cdots+k_{a-1}\right) \cdots k_{a-1}}\left\{\left[\overline{\delta_{\Lambda_{1}}^{(1)} A(1)},\left[\ldots,\left[A(a-1), \widetilde{\delta}_{\Lambda_{2}}^{(n-a)} A_{\mu}(a)\right] \ldots\right]\right]+\ldots\right. \\
& \left.+\left[A(1),\left[\ldots,\left[\overline{\delta_{\Lambda_{1}}^{(1)} A(a-1)}, \widetilde{\delta}_{\Lambda_{2}}^{(n-a)} A_{\mu}(a)\right] \ldots\right]\right]\right\} \\
& +\sum_{a=2}^{n-1} \frac{(-1)^{a}\left(k_{1}+\cdots+k_{a-1}\right)_{\mu}}{\left(k_{1}+\cdots+k_{a-1}\right) \cdots k_{a-1} k_{a}}\left\{\left[\overline{\delta_{\Lambda_{1}}^{(1)} A(1)},\left[\ldots,\left[A(a-1), \widetilde{\delta}_{\Lambda_{2}}^{(n-a)} A(a)\right] \ldots\right]\right]+\cdots\right. \\
& \left.+\left[A(1),\left[\ldots,\left[\overline{\delta_{\Lambda_{1}}^{(1)} A(a-1)}, \widetilde{\delta}_{\Lambda_{2}}^{(n-a)} A(a)\right] \ldots\right]\right]\right\} \\
& -\sum_{a=2}^{n-1} \frac{(-1)^{a}\left[\overline{\delta_{\Lambda_{1}}^{(1)} A_{\mu}(1)},\left[A(2),\left[\ldots,\left[A(a-1), \widetilde{\delta}_{\Lambda_{2}}^{(n-a)} A(a)\right] \cdots\right]\right]\right]}{\left(k_{2}+\cdots+k_{a-1}\right) \cdots k_{a-1} k_{a}} \\
& -\sum_{a=3}^{n-1} \frac{(-1)^{a}}{\left(k_{2}+\cdots+k_{a-1}\right) \cdots k_{a-1} k_{a}}\left\{\left[A_{\mu}(1),\left[\overline{\delta_{\Lambda_{1}}^{(1)} A(2)},\left[\ldots,\left[A(a-1), \widetilde{\delta}_{\Lambda_{2}}^{(n-a)} A(a)\right] \ldots\right]\right]\right]+\cdots\right. \\
& \left.+\left[A_{\mu}(1),\left[A(2),\left[\ldots,\left[\overline{\delta_{\Lambda_{1}}^{(1)} A(a-1)}, \widetilde{\delta}_{\Lambda_{2}}^{(n-a)} A(a)\right] \ldots\right]\right]\right]\right\} .
\end{aligned}
$$

Also, from Eq. (35), we derive 


$$
\begin{aligned}
- & \widetilde{\delta}_{\widetilde{L}^{(n)}}^{(0)} A_{\mu}(k) \\
= & -\imath k_{\mu} \widetilde{L}^{(n)}(k) \\
= & \sum_{a=1}^{n-1} \frac{(-1)^{a}\left(k_{1}+\cdots+k_{a}\right)_{\mu}}{\left(k_{1}+\cdots+k_{a}\right) \cdots k_{a} k_{a+1}}\left[A(1),\left[\ldots,\left[A(a), \widetilde{\delta}_{\widetilde{L}^{(n-a)}}^{(0)} A(a+1)\right] \ldots\right]\right] \\
& +\sum_{a=1}^{n-1} \frac{(-1)^{a}}{\left(k_{1}+\cdots+k_{a}\right) \cdots k_{a}}\left[A(1),\left[\ldots,\left[A(a), \widetilde{\delta}_{\widetilde{L}^{(n-a)}}^{(0)} A_{\mu}(a+1)\right] \ldots\right]\right] \\
& -\sum_{a=0}^{n-3} \sum_{\ell=1}^{n-a-2} \frac{(-1)^{a}\left(k_{1}+\cdots+k_{a+2}\right)_{\mu}}{2\left(k_{1}+\cdots+k_{a}\right) \cdots k_{a} k_{a+1} k_{a+2}}\left[A(1),\left[\ldots,\left[A(a),\left[\widetilde{\delta}_{\Lambda_{1}}^{(\ell)} A(a+1), \widetilde{\delta}_{\Lambda_{2}}^{(n-\ell-a-1)} A(a+2)\right]\right] \ldots\right]\right] .
\end{aligned}
$$

Adding the results of Eqs. (42) and (43), we obtain

$$
\begin{aligned}
& \left(\delta_{\Lambda_{2}}^{(0)} \widetilde{\delta}_{\Lambda_{1}}^{(n)}-\widetilde{\delta}_{\widetilde{L}^{(n)}}^{(0)}\right) A_{\mu}(k) \\
& ={ }_{l}\left[\Lambda_{1}(1), R(2) \delta_{\Lambda_{2}}^{(n-1)} A_{\mu}(2)\right] \\
& -\sum_{a=1}^{n-1} \frac{(-1)^{a}}{\left(k_{1}+\cdots+k_{a}\right) \cdots k_{a}}\left[A(1),\left[\ldots,\left[A(a),\left(\delta_{\Lambda_{2}}^{(0)} \widetilde{\delta}_{\Lambda_{1}}^{(n-a)}-\widetilde{\delta}_{\widetilde{L}^{(n-a)}}^{(0)}\right) A_{\mu}(a+1)\right] \ldots\right]\right] \\
& -\sum_{a=1}^{n-1} \frac{(-1)^{a}\left(k_{1}+\cdots+k_{a}\right)_{\mu}}{\left(k_{1}+\cdots+k_{a}\right) \cdots k_{a} k_{a+1}}\left[A(1),\left[\ldots,\left[A(a),\left(\delta_{\Lambda_{2}}^{(0)} \widetilde{\delta}_{\Lambda_{1}}^{(n-a)}-\widetilde{\delta}_{\widetilde{L}^{(n-a)}}^{(0)}\right) A(a+1)\right] \ldots\right]\right] \\
& +\sum_{a=1}^{n-1} \frac{(-1)^{a}}{\left(k_{2}+\cdots+k_{a}\right) \cdots k_{a} k_{a+1}}\left[A_{\mu}(1),\left[\ldots,\left[A(a), \delta_{\Lambda_{2}}^{(0)} \widetilde{\delta}_{\Lambda_{1}}^{(n-a)} A(a+1)\right] \ldots\right]\right] \\
& +\sum_{a=2}^{n-1} \frac{(-1)^{a}}{\left(k_{1}+\cdots+k_{a-1}\right) \cdots k_{a-1}}\left\{\left[\overline{\delta_{\Lambda_{1}}^{(1)} A(1)},\left[\ldots,\left[A(a-1), \widetilde{\delta}_{\Lambda_{2}}^{(n-a)} A_{\mu}(a)\right] \ldots\right]\right]+\cdots\right. \\
& \left.+\left[A(1),\left[\ldots,\left[\overline{\delta_{\Lambda_{1}}^{(1)} A(a-1)}, \widetilde{\delta}_{\Lambda_{2}}^{(n-a)} A_{\mu}(a)\right] \ldots\right]\right]\right\} \\
& +\sum_{a=2}^{n-1} \frac{(-1)^{a}\left(k_{1}+\cdots+k_{a-1}\right)_{\mu}}{\left(k_{1}+\cdots+k_{a-1}\right) \cdots k_{a-1} k_{a}}\left\{\left[\overline{\delta_{\Lambda_{1}}^{(1)} A(1)},\left[\ldots,\left[A(a-1), \widetilde{\delta}_{\Lambda_{2}}^{(n-a)} A(a)\right] \ldots\right]\right]+\cdots\right. \\
& \left.+\left[A(1),\left[\ldots,\left[\overline{\delta_{\Lambda_{1}}^{(1)} A(a-1)}, \widetilde{\delta}_{\Lambda_{2}}^{(n-a)} A(a)\right] \cdots\right]\right]\right\} \\
& -\sum_{a=2}^{n-1} \frac{(-1)^{a}\left[\overline{\delta_{\Lambda_{1}}^{(1)} A_{\mu}(1)},\left[A(2),\left[\ldots,\left[A(a-1), \widetilde{\delta}_{\Lambda_{2}}^{(n-a)} A(a)\right] \cdots\right]\right]\right]}{\left(k_{2}+\cdots+k_{a-1}\right) \cdots k_{a-1} k_{a}} \\
& -\sum_{a=3}^{n-1} \frac{(-1)^{a}}{\left(k_{2}+\cdots+k_{a-1}\right) \cdots k_{a-1} k_{a}}\left\{\left[A_{\mu}(1),\left[\overline{\delta_{\Lambda_{1}}^{(1)} A(2)},\left[\ldots,\left[A(a-1), \widetilde{\delta}_{\Lambda_{2}}^{(n-a)} A(a)\right] \cdots\right]\right]\right]+\cdots\right. \\
& \left.+\left[A_{\mu}(1),\left[A(2),\left[\ldots,\left[\overline{\delta_{\Lambda_{1}}^{(1)} A(a-1)}, \widetilde{\delta}_{\Lambda_{2}}^{(n-a)} A(a)\right] \cdots\right]\right]\right]\right\} \\
& -\sum_{a=0}^{n-3} \sum_{\ell=1}^{n-a-2} \frac{(-1)^{a}\left(k_{1}+\cdots+k_{a+2}\right)_{\mu}}{2\left(k_{1}+\cdots+k_{a}\right) \cdots k_{a} k_{a+1} k_{a+2}}\left[A(1),\left[\ldots,\left[A(a),\left[\widetilde{\delta}_{\Lambda_{1}}^{()} A(a+1), \widetilde{\delta}_{\Lambda_{2}}^{(n-\ell-a-1)} A(a+2)\right]\right] \ldots\right]\right] .
\end{aligned}
$$

From Eq. (34), we obtain

$$
\begin{aligned}
\sum_{\ell=1}^{n-2} & \delta_{\Lambda_{2}}^{(\ell)} \widetilde{\delta}_{\Lambda_{1}}^{(n-\ell)} A_{\mu}(k) \\
& =\sum_{\ell=1}^{n-2} \sum_{a=1}^{n-\ell-1} \frac{(-1)^{a}}{\left(k_{1}+\cdots+k_{a}\right) \cdots k_{a}}\left\{\left[R(1) \delta_{\Lambda_{1}}^{(\ell)} A(1),\left[\ldots,\left[A(a), \widetilde{\delta}_{\Lambda_{2}}^{(n-\ell-a)} A_{\mu}(a+1)\right] \ldots\right]\right]+\ldots\right.
\end{aligned}
$$




$$
\begin{aligned}
& \left.+\left[A(1),\left[\ldots,\left[R(a) \delta_{\Lambda_{1}}^{(\ell)} A(a), \widetilde{\delta}_{\Lambda_{2}}^{(n-\ell-a)} A_{\mu}(a+1)\right] \ldots\right]\right]\right\} \\
& -\sum_{\ell=1}^{n-2} \sum_{a=1}^{n-\ell-1} \frac{(-1)^{a}}{\left(k_{1}+\cdots+k_{a}\right) \cdots k_{a}}\left[A(1),\left[\ldots,\left[A(a), \delta_{\Lambda_{2}}^{(\ell)} \widetilde{\delta}_{\Lambda_{1}}^{(n-\ell-a)} A_{\mu}(a+1)\right] \ldots\right]\right] \\
& +\sum_{\ell=1}^{n-2} \sum_{a=1}^{n-\ell-1} \frac{(-1)^{a}\left(k_{1}+\cdots+k_{a}\right)_{\mu}}{\left(k_{1}+\cdots+k_{a}\right) \cdots k_{a} k_{a+1}}\left\{\left[R(1) \delta_{\Lambda_{1}}^{(\ell)} A(1),\left[\ldots,\left[A(a), \widetilde{\delta}_{\Lambda_{2}}^{(n-\ell-a)} A(a+1)\right] \ldots\right]\right]+\ldots\right. \\
& \left.+\left[A(1),\left[\ldots,\left[R(a) \delta_{\Lambda_{1}}^{(\ell)} A(a), \widetilde{\delta}_{\Lambda_{2}}^{(n-\ell-a)} A(a+1)\right] \ldots\right]\right]\right\} \\
& -\sum_{\ell=1}^{n-2} \sum_{a=1}^{n-\ell-1} \frac{(-1)^{a}\left(k_{1}+\cdots+k_{a}\right)_{\mu}}{\left(k_{1}+\cdots+k_{a}\right) \cdots k_{a} k_{a+1}}\left[A(1),\left[\ldots,\left[A(a), \delta_{\Lambda_{2}}^{(\ell)} \widetilde{\delta}_{\Lambda_{1}}^{(n-\ell-a)} A(a+1)\right] \ldots\right]\right] \\
& -\sum_{\ell=1}^{n-2} \sum_{a=1}^{n-\ell-1} \frac{(-1)^{a}\left[R(1) \delta_{\Lambda_{1}}^{(\ell)} A_{\mu}(1),\left[A(2),\left[\ldots,\left[A(a), \widetilde{\delta}_{\Lambda_{2}}^{(n-\ell-a)} A(a+1)\right] \ldots\right]\right]\right]}{\left(k_{2}+\cdots+k_{a}\right) \cdots k_{a} k_{a+1}} \\
& -\sum_{\ell=1}^{n-2} \sum_{a=1}^{n-\ell-1} \frac{(-1)^{a}}{\left(k_{2}+\cdots+k_{a}\right) \cdots k_{a} k_{a+1}}\left\{\left[A_{\mu}(1),\left[R(2) \delta_{\Lambda_{1}}^{(\ell)} A(2),\left[\ldots,\left[A(a), \widetilde{\delta}_{\Lambda_{2}}^{n-\ell-a)} A(a+1)\right] \ldots\right]\right]+\cdots\right.\right. \\
& \left.+\left[A_{\mu}(1),\left[A(2),\left[\ldots,\left[R(a) \delta_{\Lambda_{1}}^{(\ell)} A(a), \widetilde{\delta}_{\Lambda_{2}}^{(n-\ell-a)} A(a+1)\right] \ldots\right]\right]\right]\right\} \\
& +\sum_{\ell=1}^{n-2} \sum_{a=1}^{n-\ell-1} \frac{(-1)^{a}\left[A_{\mu}(1),\left[A(2),\left[\ldots,\left[A(a), \delta_{\Lambda_{2}}^{(\ell)} \widetilde{\delta}_{\Lambda_{1}}^{(n-\ell-a)} A(a+1)\right] \ldots\right]\right]\right]}{\left(k_{2}+\cdots+k_{a}\right) \cdots k_{a} k_{a+1}}
\end{aligned}
$$

while from Eq. (33), we easily derive that

$$
\delta_{\Lambda_{2}}^{(n-1)} \widetilde{\delta}_{\Lambda_{1}}^{(1)} A_{\mu}(k)=-{ }_{l}\left[\Lambda_{1}(1), R(2) \delta_{\Lambda_{2}}^{(n-1)} A_{\mu}(2)\right] .
$$

Equations (33) and (35) yield

$$
\begin{aligned}
-\widetilde{\delta}_{\widetilde{L}^{(n-1)}}^{(1)} A_{\mu}(k)= & -\sum_{a=1}^{n-1} \frac{(-1)^{a}}{\left(k_{2}+\cdots+k_{a}\right) \cdots k_{a} k_{a+1}}\left[A_{\mu}(1),\left[A(2),\left[\ldots,\left[A(a), \widetilde{\delta}_{\widetilde{L}^{(n-a)}}^{(0)} A(a+1)\right] \ldots\right]\right]\right] \\
& +\sum_{a=1}^{n-3} \sum_{\ell=1}^{n-a-2} \frac{(-1)^{a}}{2\left(k_{2}+\cdots+k_{a}\right) \cdots k_{a} k_{a+1} k_{a+2}} \\
& \times\left[A_{\mu}(1),\left[A(2),\left[\ldots,\left[A(a),\left[\widetilde{\delta}_{\Lambda_{1}}^{(\ell)} A(a+1), \widetilde{\delta}_{\Lambda_{2}}^{(n-\ell-a-1)} A(a+2)\right]\right] \ldots\right]\right]\right] .
\end{aligned}
$$

The last term in Eq. (39) to be calculated reads

$$
\begin{aligned}
-\sum_{\ell=2}^{n-1} \widetilde{\delta}_{\widetilde{L}^{(n-\ell)}}^{(\ell)} A_{\mu}(k)= & \sum_{\ell=2}^{n-1} \sum_{a=1}^{\ell-1} \frac{(-1)^{a}}{\left(k_{1}+\cdots+k_{a}\right) \cdots k_{a}}\left[A(1),\left[\ldots,\left[A(a), \widetilde{\delta}_{\widetilde{L}^{(n-\ell)}}^{\ell-a)} A_{\mu}(a+1)\right] \ldots\right]\right] \\
& +\sum_{\ell=2}^{n-1} \sum_{a=1}^{\ell-1} \frac{(-1)^{a}\left(k_{1}+\cdots+k_{a}\right)_{\mu}}{\left(k_{1}+\cdots+k_{a}\right) \cdots k_{a} k_{a+1}}\left[A(1),\left[\ldots,\left[A(a), \widetilde{\delta}_{\tilde{L}^{(n-\ell)}}^{\ell-a)} A(a+1)\right] \ldots\right]\right] \\
& -\sum_{\ell=2}^{n-1} \sum_{a=1}^{\ell-1} \frac{(-1)^{a}}{\left(k_{2}+\cdots+k_{a}\right) \cdots k_{a} k_{a+1}}\left[A_{\mu}(1),\left[A(2),\left[\ldots,\left[A(a), \widetilde{\delta}_{\widetilde{L}^{(n-\ell)}}^{\ell-a)} A(a+1)\right] \ldots\right]\right]\right] .
\end{aligned}
$$

Finally, we add the results from Eqs. (44), (45), (46), (47), and (48) to obtain the LHS of Eq. (39):

$$
\begin{aligned}
& \sum_{\ell=0}^{n-1}\left(\delta_{\Lambda_{2}}^{(\ell)} \widetilde{\delta}_{\Lambda_{1}}^{(n-\ell)}-\widetilde{\delta}_{\widetilde{L}^{(n-\ell)}}^{(\ell)} A_{\mu}(k)\right. \\
& \quad=\sum_{\ell=1}^{n-2} \sum_{a=2}^{n-\ell} \frac{(-1)^{a}}{\left(k_{1}+\cdots+k_{a-1}\right) \cdots k_{a-1}}\left\{\left[\widetilde{\delta}_{\Lambda_{1}}^{\ell)} A(1),\left[\ldots,\left[A(a-1), \widetilde{\delta}_{\Lambda_{2}}^{(n-\ell-a+1)} A_{\mu}(a)\right] \ldots\right]\right]+\cdots\right.
\end{aligned}
$$




$$
\begin{aligned}
& \left.+\left[A(1),\left[\ldots,\left[\widetilde{\delta}_{\Lambda_{1}}^{(\ell)} A(a-1), \widetilde{\delta}_{\Lambda_{2}}^{(n-\ell-a+1)} A_{\mu}(a)\right] \ldots\right]\right]\right\} \\
& -\sum_{\ell=2}^{n-2} \sum_{a=2}^{n-\ell} \frac{(-1)^{a}}{\left(k_{1}+\cdots+k_{a-1}\right) \cdots k_{a-1}}\left\{\left[\overline{\delta_{\Lambda_{1}}^{(\ell)} A(1)},\left[\ldots,\left[A(a-1), \widetilde{\delta}_{\Lambda_{2}}^{(n-\ell-a+1)} A_{\mu}(a)\right] \ldots\right]\right]+\cdots\right. \\
& \left.+\left[A(1),\left[\ldots,\left[\overline{\delta_{\Lambda_{1}}^{(\ell)} A(a-1)}, \widetilde{\delta}_{\Lambda_{2}}^{(n-\ell-a+1)} A_{\mu}(a)\right] \ldots\right]\right]\right\} \\
& +\sum_{\ell=1}^{n-2} \sum_{a=2}^{n-\ell} \frac{(-1)^{a}\left(k_{1}+\cdots+k_{a-1}\right)_{\mu}}{\left(k_{1}+\cdots+k_{a-1}\right) \cdots k_{a-1} k_{a}}\left\{\left[\widetilde{\delta}_{\Lambda_{1}}^{\ell)} A(1),\left[\ldots,\left[A(a-1), \widetilde{\delta}_{\Lambda_{2}}^{(n-\ell-a+1)} A(a)\right] \ldots\right]\right]+\cdots\right. \\
& \left.+\left[A(1),\left[\ldots,\left[\widetilde{\delta}_{\Lambda_{1}}^{(\ell)} A(a-1), \widetilde{\delta}_{\Lambda_{2}}^{(n-\ell-a+1)} A(a)\right] \ldots\right]\right]\right\} \\
& -\sum_{\ell=2}^{n-2} \sum_{a=2}^{n-\ell} \frac{(-1)^{a}\left(k_{1}+\cdots+k_{a-1}\right)_{\mu}}{\left(k_{1}+\cdots+k_{a-1}\right) \cdots k_{a-1} k_{a}}\left\{\left[\overline{\delta_{\Lambda_{1}}^{(\ell)} A(1)},\left[\ldots,\left[A(a-1), \widetilde{\delta}_{\Lambda_{2}}^{(n-\ell-a+1)} A(a)\right] \ldots\right]\right]+\cdots\right. \\
& \left.+\left[A(1),\left[\ldots,\left[\overline{\delta_{\Lambda_{1}}^{(\ell)} A(a-1)}, \widetilde{\delta}_{\Lambda_{2}}^{(n-\ell-a+1)} A(a)\right] \ldots\right]\right]\right\} \\
& -\sum_{\ell=1}^{n-2} \sum_{a=2}^{n-\ell} \frac{(-1)^{a}\left[\widetilde{\delta}_{\Lambda_{1}}^{\ell} A_{\mu}(1),\left[A(2),\left[\ldots,\left[A(a-1), \widetilde{\delta}_{\Lambda_{2}}^{(n-\ell-a+1)} A(a)\right] \ldots\right]\right]\right]}{\left(k_{2}+\cdots+k_{a-1}\right) \cdots k_{a-1} k_{a}} \\
& +\sum_{\ell=2}^{n-2} \sum_{a=2}^{n-\ell} \frac{(-1)^{a}\left[\overline{\delta_{\Lambda_{1}}^{(\ell} A_{\mu}(1)},\left[A(2),\left[\ldots,\left[A(a-1), \widetilde{\delta}_{\Lambda_{2}}^{(n-\ell-a+1)} A(a)\right] \ldots\right]\right]\right]}{\left(k_{2}+\cdots+k_{a-1}\right) \cdots k_{a-1} k_{a}} \\
& -\sum_{\ell=1}^{n-3} \sum_{a=3}^{n-\ell} \frac{(-1)^{a}}{\left(k_{2}+\cdots+k_{a-1}\right) \cdots k_{a-1} k_{a}}\left\{\left[A_{\mu}(1),\left[\widetilde{\delta}_{\Lambda_{1}}^{(\ell)} A(2),\left[\ldots,\left[A(a-1), \widetilde{\delta}_{\Lambda_{2}}^{(n-\ell-a+1)} A(a)\right] \ldots\right]\right]\right]+\cdots\right. \\
& \left.+\left[A_{\mu}(1),\left[A(2),\left[\ldots,\left[\widetilde{\delta}_{\Lambda_{1}}^{(\bigcap)} A(a-1), \widetilde{\delta}_{\Lambda_{2}}^{(n-\ell-a+1)} A(a)\right] \ldots\right]\right]\right]\right\} \\
& +\sum_{\ell=2}^{n-3} \sum_{a=3}^{n-\ell} \frac{(-1)^{a}}{\left(k_{2}+\cdots+k_{a-1}\right) \cdots k_{a-1} k_{a}}\left\{\left[A_{\mu}(1),\left[\overline{\delta_{\Lambda_{1}}^{(\ell)} A(2)},\left[\ldots,\left[A(a-1), \widetilde{\delta}_{\Lambda_{2}}^{(n-\ell-a+1)} A(a)\right] \ldots\right]\right]\right]+\cdots\right. \\
& \left.+\left[A_{\mu}(1),\left[A(2),\left[\ldots,\left[\overline{\delta_{\Lambda_{1}}^{(\ell)} A(a-1)}, \widetilde{\delta}_{\Lambda_{2}}^{(n-\ell-a+1)} A(a)\right] \ldots\right]\right]\right]\right\} \\
& -\sum_{\ell=1}^{n-2} \sum_{a=2}^{n-\ell} \frac{(-1)^{a}\left(k_{1}+\cdots+k_{a}\right)_{\mu}}{2\left(k_{1}+\cdots+k_{a-2}\right) \cdots k_{a-2} k_{a-1} k_{a}}\left[A(1),\left[\ldots,\left[A(a-2),\left[\widetilde{\delta}_{\Lambda_{1}}^{\ell} A(a-1), \widetilde{\delta}_{\Lambda_{2}}^{(n-\ell-a+1)} A(a)\right]\right] \ldots\right]\right] \\
& \left.+\sum_{\ell=1}^{n-3} \sum_{a=3}^{n-\ell} \frac{(-1)^{a}}{2\left(k_{2}+\cdots+k_{a-2}\right) \cdots k_{a-2} k_{a-1} k_{a}}\left[A_{\mu}(1),\left[A(2),\left[\ldots,\left[A(a-2),\left[\widetilde{\delta}_{\Lambda_{1}}^{(}\right) A(a-1), \widetilde{\delta}_{\Lambda_{2}}^{(n-\ell-a+1)} A(a)\right]\right] \ldots\right]\right]\right] .
\end{aligned}
$$

In obtaining the result of Eq. (49), we made use of the lower order relation of Eq. (40) to eliminate certain terms.

Expression (49) can be written in a more compact form by introducing an extra summation index $b$, which labels the position of $\overline{\delta_{\Lambda}^{(\ell)} A}$ or $\widetilde{\delta}_{\Lambda}^{(\ell)} A$ in the commutator. We then have

$$
\begin{aligned}
\sum_{\ell=0}^{n-1}( & \delta_{\Lambda_{2}}^{(\ell)} \widetilde{\delta}_{\Lambda_{1}}^{(n-\ell)}-\widetilde{\delta}_{\widetilde{L}^{(n-\ell)}(\ell) A_{\mu}}^{(k)} \\
= & \sum_{\ell=1}^{n-2} \sum_{a=2}^{n-\ell} \sum_{b=1}^{a-1} \frac{(-1)^{a}}{\left(k_{1}+\cdots+k_{a-1}\right) \cdots k_{a-1}}\left[A(1),\left[\ldots,\left[\widetilde{\delta}_{\Lambda_{1}}^{(\ell)} A(b),\left[\ldots,\left[A(a-1), \widetilde{\delta}_{\Lambda_{2}}^{(n-\ell-a+1)} A_{\mu}(a)\right] \ldots\right]\right] \ldots\right]\right] \\
& -\sum_{\ell=2}^{n-2} \sum_{a=2}^{n-\ell} \sum_{b=1}^{a-1} \frac{(-1)^{a}}{\left(k_{1}+\cdots+k_{a-1}\right) \cdots k_{a-1}}\left[A(1),\left[\ldots,\left[\overline{\delta_{\Lambda_{1}}^{(\ell)} A(b)},\left[\ldots,\left[A(a-1), \widetilde{\delta}_{\Lambda_{2}}^{(n-\ell-a+1)} A_{\mu}(a)\right] \ldots\right]\right] \ldots\right]\right]
\end{aligned}
$$




$$
\begin{aligned}
& +\sum_{\ell=1}^{n-2} \sum_{a=2}^{n-\ell} \sum_{b=1}^{a-1} \frac{(-1)^{a}\left(k_{1}+\cdots+k_{a-1}\right)_{\mu}}{\left(k_{1}+\cdots+k_{a-1}\right) \cdots k_{a-1} k_{a}}\left[A(1),\left[\ldots,\left[\widetilde{\delta}_{\Lambda_{1}}^{(\ell)} A(b),\left[\ldots,\left[A(a-1), \widetilde{\delta}_{\Lambda_{2}}^{(n-\ell-a+1)} A(a)\right] \ldots\right]\right] \ldots\right]\right] \\
& -\sum_{\ell=2}^{n-2} \sum_{a=2}^{n-\ell} \sum_{b=1}^{a-1} \frac{(-1)^{a}\left(k_{1}+\cdots+k_{a-1}\right)_{\mu}}{\left(k_{1}+\cdots+k_{a-1}\right) \cdots k_{a-1} k_{a}}\left[A(1),\left[\ldots,\left[\overline{\delta_{\Lambda_{1}}^{(\ell)} A(b)},\left[\ldots,\left[A(a-1), \widetilde{\delta}_{\Lambda_{2}}^{(n-\ell-a+1)} A(a)\right] \ldots\right]\right] \ldots\right]\right] \\
& -\sum_{\ell=1}^{n-2} \sum_{a=2}^{n-\ell} \frac{(-1)^{a}\left[\widetilde{\delta}_{\Lambda_{1}}^{\ell \ell} A_{\mu}(1),\left[A(2),\left[\ldots,\left[A(a-1), \widetilde{\delta}_{\Lambda_{2}}^{(n-\ell-a+1)} A(a)\right] \ldots\right]\right]\right]}{\left(k_{2}+\cdots+k_{a-1}\right) \cdots k_{a-1} k_{a}} \\
& +\sum_{\ell=2}^{n-2} \sum_{a=2}^{n-\ell} \frac{(-1)^{a}\left[\overline{\delta_{\Lambda_{1}}^{(\ell)} A_{\mu}(1)},\left[A(2),\left[\ldots,\left[A(a-1), \widetilde{\delta}_{\Lambda_{2}}^{(n-\ell-a+1)} A(a)\right] \ldots\right]\right]\right]}{\left(k_{2}+\cdots+k_{a-1}\right) \cdots k_{a-1} k_{a}} \\
& -\sum_{\ell=1}^{n-3} \sum_{a=3}^{n-\ell} \sum_{b=2}^{a-1} \frac{(-1)^{a}}{\left(k_{2}+\cdots+k_{a-1}\right) \cdots k_{a-1} k_{a}}\left[A_{\mu}(1),\left[\ldots,\left[\widetilde{\delta}_{\Lambda_{1}}^{()} A(b),\left[\ldots,\left[A(a-1), \widetilde{\delta}_{\Lambda_{2}}^{(n-\ell-a+1)} A(a)\right] \ldots\right]\right] \ldots\right]\right] \\
& +\sum_{\ell=2}^{n-3} \sum_{a=3}^{n-\ell} \sum_{b=2}^{a-1} \frac{(-1)^{a}}{\left(k_{2}+\cdots+k_{a-1}\right) \cdots k_{a-1} k_{a}}\left[A_{\mu}(1),\left[\ldots,\left[\overline{\delta_{\Lambda_{1}}^{(\ell)} A(b)},\left[\ldots,\left[A(a-1), \widetilde{\delta}_{\Lambda_{2}}^{(n-\ell-a+1)} A(a)\right] \ldots\right]\right] \ldots\right]\right] \\
& -\sum_{\ell=1}^{n-2} \sum_{a=2}^{n-\ell} \frac{(-1)^{a}\left(k_{1}+\cdots+k_{a}\right)_{\mu}}{2\left(k_{1}+\cdots+k_{a-2}\right) \cdots k_{a-2} k_{a-1} k_{a}}\left[A(1),\left[\ldots,\left[A(a-2),\left[\widetilde{\delta}_{\Lambda_{1}}^{\ell} A(a-1), \widetilde{\delta}_{\Lambda_{2}}^{(n-\ell-a+1)} A(a)\right]\right]\right]\right] \\
& +\sum_{\ell=1}^{n-3} \sum_{a=3}^{n-\ell} \frac{(-1)^{a}}{2\left(k_{2}+\cdots+k_{a-2}\right) \cdots k_{a-2} k_{a-1} k_{a}}\left[A_{\mu}(1),\left[A(2),\left[\ldots,\left[A(a-2),\left[\widetilde{\delta}_{\Lambda_{1}}^{(\ell)} A(a-1), \widetilde{\delta}_{\Lambda_{2}}^{(n-\ell-a+1)} A(a)\right]\right] \cdots\right]\right]\right] .
\end{aligned}
$$

Our aim is to prove that the right-hand side (RHS) of Eq. (50) vanishes. There are three types of terms in expression (50), depending on the position of the index $\mu$ : the index can appear in the combination $\delta_{\Lambda}^{(i)} A_{\mu}(j)$, it can appear in the combination $A_{\mu}(i)$ without a $\delta_{\Lambda}^{(j)}$ acting on this $A_{\mu}(i)$, or it can appear in the combination $k_{i \mu}$. The quantities $i$ and $j$ can have any value. For the expression (50) to vanish, the three types of terms have to vanish separately. The three types of terms will be respectively denoted by $X_{1}, X_{2}$, and $X_{3}$, and we now show that they indeed vanish.

\section{A. $X_{1}$}

We start by showing that the terms in Eq. (50) with a $\delta_{\Lambda}^{(i)} A_{\mu}(j)$ combination vanish. They are

$$
\begin{aligned}
X_{1}= & \sum_{\ell=1}^{n-2} \sum_{a=2}^{n-\ell} \sum_{b=1}^{a-1} \frac{(-1)^{a}}{\left(k_{1}+\cdots+k_{a-1}\right) \cdots k_{a-1}}\left[A(1),\left[\ldots,\left[\widetilde{\delta}_{\Lambda_{1}}^{(\ell)} A(b),\left[\ldots,\left[A(a-1), \widetilde{\delta}_{\Lambda_{2}}^{(n-\ell-a+1)} A_{\mu}(a)\right] \ldots\right]\right] \ldots\right]\right] \\
& -\sum_{\ell=2}^{n-2} \sum_{a=2}^{n-\ell} \sum_{b=1}^{a-1} \frac{(-1)^{a}}{\left(k_{1}+\cdots+k_{a-1}\right) \ldots k_{a-1}}\left[A(1),\left[\ldots,\left[\overline{\left.\left.\left.\delta_{\Lambda_{1}}^{(\ell)} A(b),\left[\ldots,\left[A(a-1), \widetilde{\delta}_{\Lambda_{2}}^{(n-\ell-a+1)} A_{\mu}(a)\right] \ldots\right]\right] \ldots\right]\right]}\right.\right.\right. \\
& -\sum_{\ell=1}^{n-2} \sum_{a=2}^{n-\ell} \frac{(-1)^{a}\left[\widetilde{\delta}_{\Lambda_{1}}^{(\ell)} A_{\mu}(1),\left[A(2),\left[\ldots,\left[A(a-1), \widetilde{\delta}_{\Lambda_{2}}^{(n-\ell-a+1)} A(a)\right] \ldots\right]\right]\right]}{\left(k_{2}+\cdots+k_{a-1}\right) \ldots k_{a-1} k_{a}} \\
& +\sum_{\ell=2}^{n-2} \sum_{a=2}^{n-\ell} \frac{(-1)^{a}\left[\overline{\left.\overline{\delta_{\Lambda_{1}}^{(\ell)} A_{\mu}(1)},\left[A(2),\left[\ldots,\left[A(a-1), \widetilde{\delta}_{\Lambda_{2}}^{(n-\ell-a+1)} A(a)\right] \ldots\right]\right]\right]}\right.}{\left(k_{2}+\cdots+k_{a-1}\right) \cdots k_{a-1} k_{a}}
\end{aligned}
$$

where $\delta_{\Lambda_{1}}^{\overline{\left(\bar{C}_{\mu}\right.}}$ denotes the term in its expansion of Eq. (34) proportional to $\widetilde{\delta}_{\Lambda_{1}}^{(\ell-c)} A_{\mu}$, i.e.,

$$
\overline{\overline{\delta_{\Lambda_{1}}^{(\ell)} A_{\mu}(k)}}=-\sum_{c=1}^{\ell-1} \frac{(-1)^{c}}{\left(k_{1}+\cdots+k_{c}\right) \ldots k_{c}}\left[A(1),\left[\ldots,\left[A(c), \widetilde{\delta}_{\Lambda_{1}}^{\ell-c)} A_{\mu}(c+1)\right] \ldots\right]\right] .
$$

We shall treat the first two terms and the last two terms in expression (51) for $X_{1}$ separately. We then have that 


$$
X_{1}=X_{1 A}+X_{1 B}
$$

where

$$
\begin{aligned}
X_{1 A}= & \sum_{\ell=1}^{n-2} \sum_{a=2}^{n-\ell} \sum_{b=1}^{a-1} \frac{(-1)^{a}}{\left(k_{1}+\cdots+k_{a-1}\right) \cdots k_{a-1}}\left[A(1),\left[\ldots,\left[\widetilde{\delta}_{\Lambda_{1}}^{\ell)} A(b),\left[\ldots,\left[A(a-1), \widetilde{\delta}_{\Lambda_{2}}^{(n-\ell-a+1)} A_{\mu}(a)\right] \ldots\right]\right] \ldots\right]\right] \\
& -\sum_{\ell=2}^{n-2} \sum_{a=2}^{n-\ell} \sum_{b=1}^{a-1} \frac{(-1)^{a}}{\left(k_{1}+\cdots+k_{a-1}\right) \cdots k_{a-1}}\left[A(1),\left[\ldots,\left[\overline{\delta_{\Lambda_{1}}^{(\ell)} A(b)},\left[\ldots,\left[A(a-1), \widetilde{\delta}_{\Lambda_{2}}^{(n-\ell-a+1)} A_{\mu}(a)\right] \ldots\right]\right] \ldots\right]\right],
\end{aligned}
$$

and

$$
\begin{aligned}
X_{1 B}= & -\sum_{\ell=1}^{n-2} \sum_{a=2}^{n-\ell} \frac{(-1)^{a}\left[\widetilde{\delta}_{\Lambda_{1}}^{(\ell)} A_{\mu}(1),\left[A(2),\left[\ldots,\left[A(a-1), \widetilde{\delta}_{\Lambda_{2}}^{(n-\ell-a+1)} A(a)\right] \ldots\right]\right]\right]}{\left(k_{2}+\cdots+k_{a-1}\right) \cdots k_{a-1} k_{a}} \\
& +\sum_{\ell=2}^{n-2} \sum_{a=2}^{n-\ell} \frac{\overline{(-1)^{a}\left[\overline{\delta_{\Lambda_{1}^{(\ell)} A_{\mu}(1)}},\left[A(2),\left[\cdots,\left[A(a-1), \widetilde{\delta}_{\Lambda_{2}}^{(n-\ell-a+1)} A(a)\right] \cdots\right]\right]\right.}}{\left(k_{2}+\cdots+k_{a-1}\right) \cdots k_{a-1} k_{a}} .
\end{aligned}
$$

We first rewrite expression $X_{1 A}$ in Eq. (54) using the expansion for $\overline{\delta_{\Lambda_{1}}^{(\varrho)} A(b)}$ given by Eq. (41):

$$
\begin{aligned}
X_{1 A}= & \sum_{\ell=1}^{n-2} \sum_{a=2}^{n-\ell} \sum_{b=1}^{a-1} \frac{(-1)^{a}}{\left(k_{1}+\cdots+k_{a-1}\right) \cdots k_{a-1}}\left[A(1),\left[\ldots,\left[\widetilde{\delta}_{\Lambda_{1}}^{\ell)} A(b),\left[\ldots,\left[A(a-1), \widetilde{\delta}_{\Lambda_{2}}^{(n-\ell-a+1)} A_{\mu}(a)\right] \ldots\right]\right] \ldots\right]\right] \\
& +\sum_{\ell=1}^{n-2} \sum_{a=2}^{n-\ell} \sum_{b=1}^{a-1} \sum_{c=1}^{\ell-1}(-1)^{a+c} \frac{\left[A(1),\left[\ldots,\left[\left[A(b),\left[\ldots,\left[A(c+b-1), \widetilde{\delta}_{\Lambda_{1}}^{(\ell-c)} A(c+b)\right] \ldots\right]\right]\right.\right.\right.}{\left(k_{1}+\cdots+k_{c+a-1}\right) \cdots\left(k_{b}+\cdots+k_{c+a-1}\right)} \\
& \frac{\left.\left.\left.\left[A(c+b+1),\left[\ldots,\left[A(c+a-1), \widetilde{\delta}_{\Lambda_{2}}^{(n-\ell-a+1)} A_{\mu}(c+a)\right] \ldots\right]\right]\right] \ldots\right]\right]}{\left(k_{b}+\cdots+k_{c+b-1}\right) \cdots k_{c+b-1}\left(k_{c+b+1}+\cdots+k_{c+a-1}\right) \cdots k_{c+a-1}}
\end{aligned}
$$

Note that, in the second term of Eq. (56), we have extended the summation over $\ell$ to include the term $\ell=1$, because it vanishes due to our summation convention (36) for the sum over $c$. The two terms in Eq. (56) can now be combined using Eq. (37):

$$
\begin{aligned}
X_{1 A}= & \sum_{\ell=1}^{n-2} \sum_{a=2}^{n-\ell} \sum_{b=1}^{a-1} \sum_{c=0}^{\ell-1}(-1)^{a+c} \frac{\left[A(1),\left[\ldots,\left[\left[A(b),\left[\ldots,\left[A(c+b-1), \widetilde{\delta}_{\Lambda_{1}}^{(\ell-c)} A(c+b)\right] \ldots\right]\right],\right.\right.\right.}{\left(k_{1}+\cdots+k_{c+a-1}\right) \cdots\left(k_{b}+\cdots+k_{c+a-1}\right)} \\
& \frac{\left.\left.\left[A(c+b+1),\left[\ldots,\left[A(c+a-1), \widetilde{\delta}_{\Lambda_{2}}^{(n-\ell-a+1)} A_{\mu}(c+a) \cdots\right]\right]\right] \ldots\right]\right]}{\left(k_{b}+\cdots+k_{c+b-1}\right) \cdots k_{c+b-1}\left(k_{c+b+1}+\cdots+k_{c+a-1}\right) \cdots k_{c+a-1}} .
\end{aligned}
$$

Using the formula (A1) in Appendix A, we can rewrite this expression (57) in the form of a nested commutator. To see how this can be done, we rename a series of variables in Eq. (57). Let

$$
k_{b}=K_{1}, \quad k_{c+b}=K_{c+1}, \quad k_{c+a}=K_{c+a-b+1},
$$

and

$$
\ell-c=P, \quad c+1=N-L-1, \quad c+a-b+1=N-1, \quad n-\ell-a+1=Q,
$$

and let us rearrange the quadruple sum in Eq. (57) in the following way: 


$$
\begin{aligned}
& \sum_{\ell=1}^{n-2} \sum_{a=2}^{n-\ell} \sum_{b=1}^{a-1} \sum_{c=0}^{\ell-1}=\sum_{\ell=1}^{n-2} \sum_{b=1}^{n-\ell} \sum_{a=b+1}^{n} \sum_{c=0}^{n-1}=\sum_{b=1}^{n-2} \sum_{\ell=1}^{n-b-1} \sum_{a=b+1}^{n-\ell} \sum_{c=0}^{\ell-1}=\sum_{b=1}^{n-2} \sum_{\ell=1}^{n-b} \sum_{a=b+1} \sum_{P=1}=\sum_{b=1}^{n-1} \sum_{\ell=1}^{n-b-1} \sum_{P=1}^{\ell} \sum_{a=b+1}^{n-\ell} \\
& =\sum_{b=1}^{n-2} \sum_{P=1}^{n-b-1} \sum_{\ell=P} \sum_{a=b+1}^{n-b-1}=\sum_{b=1}^{n-\ell} \sum_{P=1}^{n} \sum_{\ell=P}^{n-b} \sum_{L=1}^{n-1}=\sum_{b=1}^{n} \sum_{P=1}^{n-b-1} \sum_{L=1}^{n-\ell} \sum_{\ell=P}^{n-b} \\
& =\sum_{b=1}^{n-2} \sum_{P=1}^{n-b-1} \sum_{L=1}^{n-P-b} \sum_{Q=1}^{n-b-L-P+1}=\sum_{b=1}^{n-2} \sum_{P=1}^{n-b-1} \sum_{Q=1}^{n-b-P} \sum_{L=1}^{n-b-P-Q+1}=\sum_{b=1}^{n-2} \sum_{P=1}^{n-b-1} \sum_{Q=1}^{n} \sum_{L=1}^{n-b-P} .
\end{aligned}
$$

The preceding manipulations then lead to

$$
\begin{aligned}
X_{1 A}= & \sum_{b=1}^{n-2} \sum_{P=1}^{n-b-1} \sum_{Q=1}^{n-b-P} \sum_{L=1}^{N-2}(-1)^{n-P-Q+1} \frac{\left[A(1),\left[\ldots,\left[A(b-1),\left[\left[\bar{A}(1),\left[\cdots,\left[\bar{A}(N-L-2), \widetilde{\delta}_{\Lambda_{1}}^{(P)} \bar{A}(N-L-1)\right] \ldots\right]\right],\right.\right.\right.\right.}{\left(k_{1}+\cdots+K_{N-2}\right) \cdots\left(K_{1}+\cdots+K_{N-2}\right)} \\
& \frac{\left.\left.\left.\left.\left[\bar{A}(N-L),\left[\cdots,\left[\bar{A}(N-2), \widetilde{\delta}_{\Lambda_{2}}^{(Q)} \bar{A}_{\mu}(N-1)\right] \ldots\right]\right]\right]\right] \ldots\right]\right]}{\left(K_{1}+\cdots+K_{N-L-2}\right) \cdots K_{N-L-2}\left(K_{N-L}+\cdots+K_{N-2}\right) \cdots K_{N-2}},
\end{aligned}
$$

where the notation $\bar{A}$ is used to indicate that the $\bar{A}$ has a $K$-type argument. The use of formula (A1) from Appendix A allows one to perform the summation over $L$ and converts $X_{1 A}$ into a nested commutator:

$$
\begin{aligned}
X_{1 A}= & \sum_{b=1}^{n-2} \sum_{P=1}^{n-b-1} \sum_{Q=1}^{n-b-P}(-1)^{n-P-Q+1} \frac{[A(1),[\ldots,[A(b-1),[\bar{A}(1),}{\left(k_{1}+\cdots+K_{N-2}\right) \cdots\left(K_{1}+\cdots+K_{N-2}\right)} \\
& \frac{\left.\left.\left.\left.\left[\ldots,\left[\widetilde{\delta}_{\Lambda_{1}}^{(P)} \bar{A}(N-2), \widetilde{\delta}_{\Lambda_{2}}^{(Q)} \bar{A}_{\mu}(N-1)\right] \ldots \ldots\right]\right]\right] \ldots\right]\right]}{\left(K_{1}+\cdots+K_{N-3}\right) \cdots K_{N-3}} \\
= & \sum_{b=1}^{n-2} \sum_{P=1}^{n-b-1} \sum_{Q=1}^{n-b-P}(-1)^{n-P-Q+1} \frac{[A(1),[\ldots,}{\left(k_{1}+\cdots+k_{n-P-Q}\right) \cdots\left(k_{b}+\cdots+k_{n-P-Q}\right)} \\
& \frac{\left.\left[A(n-P-Q-1),\left[\widetilde{\delta}_{\Lambda_{1}}^{(P)} A(n-P-Q), \widetilde{\delta}_{\Lambda_{2}}^{(Q)} A_{\mu}(n-P-Q+1)\right] \ldots\right]\right]}{\left(k_{b}+\cdots+k_{n-P-Q-1}\right) \cdots k_{n-P-Q-1}},
\end{aligned}
$$

where, in the last equality, we returned to the original integration variables $k$.

In expression (62), the sum over $b$ can also be performed. To this end, we introduce the variable

$$
\alpha=n-P-Q
$$

and rearrange the triple sum as follows:

$$
\sum_{b=1}^{n-2} \sum_{P=1}^{n-b-1} \sum_{Q=1}^{n-b-P}=\sum_{P=1}^{n-2} \sum_{b=1}^{n-P-1} \sum_{Q=1}^{n-b-P}=\sum_{P=1}^{n-2} \sum_{Q=1}^{n-P-1} \sum_{b=1}^{n-P-Q}=\sum_{P=1}^{n-2} \sum_{Q=1}^{n-P-1} \sum_{b=1}^{\alpha} .
$$

Then

$$
X_{1 A}=\sum_{P=1}^{n-2} \sum_{Q=1}^{n-P-1} \sum_{b=1}^{\alpha}(-1)^{\alpha+1} \frac{\left[A(1),\left[\cdots,\left[A(\alpha-1),\left[\widetilde{\delta}_{\Lambda_{1}}^{(P)} A(\alpha), \widetilde{\delta}_{\Lambda_{2}}^{(Q)} A_{\mu}(\alpha+1)\right]\right] \ldots\right]\right]}{\left(k_{1}+\cdots+k_{\alpha}\right) \cdots\left(k_{b}+\cdots+k_{\alpha}\right)\left(k_{b}+\cdots+k_{\alpha-1}\right) \cdots k_{\alpha-1}}
$$

Formula (B1) of Appendix B can now be applied to perform to summation over $b$, and, finally,

$$
X_{1 A}=\sum_{P=1}^{n-2} \sum_{Q=1}^{n-P-1}(-1)^{n-P-Q+1} \frac{\left[A(1),\left[\ldots,\left[A(n-P-Q-1),\left[\widetilde{\delta}_{\Lambda_{1}}^{(P)} A(n-P-Q), \widetilde{\delta}_{\Lambda_{2}}^{(Q)} A_{\mu}(n-P-Q+1)\right]\right] \ldots\right]\right]}{\left(k_{1}+\cdots+k_{n-P-Q-1}\right) \cdots k_{n-P-Q-1} k_{n-P-Q}}
$$

The treatment of $X_{1 B}$ is very analogous to the one for $X_{1 A}$. We also rewrite expression (55) using the expansion for $\delta_{\Lambda}^{(\ell)} A(b)$ given by Eq. (41), which again allows us to combine the two terms in $X_{1 B}$ : 


$$
\begin{aligned}
X_{1 B}= & -\sum_{\ell=1}^{n-2} \sum_{a=2}^{n-\ell} \sum_{c=0}^{\ell-1}(-1)^{a+c} \frac{\left[\left[A(1),\left[\cdots,\left[A(c), \widetilde{\delta}_{\Lambda_{1}}^{\ell-c)} A_{\mu}(c+1)\right] \cdots\right]\right]\right.}{\left(k_{1}+\cdots+k_{c}\right) \cdots k_{c}} \\
& \frac{\left[A(c+2),\left[\ldots,\left[A(c+a-1), \widetilde{\delta}_{\Lambda_{2}}^{(n-\ell-a+1)} A(c+a)\right] \cdots\right]\right]}{\left(k_{c+2}+\cdots+k_{c+a-1}\right) \cdots k_{c+a-1} k_{c+a}} .
\end{aligned}
$$

Comparing Eq. (67) for $X_{1 B}$ with Eq. (57) for $X_{1 A}$, one sees that $X_{1 B}$ is the opposite of the $b=1$ term in $X_{1 A}$ with the interchange of $\widetilde{\delta}_{\Lambda} A \leftrightarrow \widetilde{\delta}_{\Lambda} A_{\mu}$. Therefore, Eq. (62) tells us that

$$
X_{1 B}=-\sum_{P=1}^{n-2} \sum_{Q=1}^{n-P-1}(-1)^{n-P-Q+1} \frac{\left[A(1),\left[\ldots,\left[A(n-P-Q-1),\left[\widetilde{\delta}_{\Lambda_{1}}^{(P)} A_{\mu}(n-P-Q), \widetilde{\delta}_{\Lambda_{2}}^{(Q)} A(n-P-Q+1)\right]\right] \ldots\right]\right]}{\left(k_{1}+\cdots+k_{n-P-Q-1}\right) \cdots k_{n-P-Q-1} k_{n-P-Q+1}} .
$$

Through some simple manipulations, we can cast Eq. (68) in a form which allows us to combine $X_{1 B}$ with $X_{1 A}$ :

$$
\begin{aligned}
X_{1 B} & =-\sum_{P=1}^{n-2} \sum_{Q=1}^{n-P-1}(-1)^{n-P-Q+1} \frac{\left[A(1),\left[\ldots,\left[A(n-P-Q-1),\left[\widetilde{\delta}_{\Lambda_{1}}^{(Q)} A(n-P-Q), \widetilde{\delta}_{\Lambda_{2}}^{(P)} A_{\mu}(n-P-Q+1)\right]\right] \ldots\right]\right]}{\left(k_{1}+\cdots+k_{n-P-Q-1}\right) \cdots k_{n-P-Q-1} k_{n-P-Q}} \\
& =-\sum_{Q=1}^{n-2} \sum_{P=1}^{n-Q-1}(-1)^{n-P-Q+1} \frac{\left[A(1),\left[\ldots,\left[A(n-P-Q-1),\left[\widetilde{\delta}_{\Lambda_{1}}^{(P)} A(n-P-Q), \widetilde{\delta}_{\Lambda_{2}}^{(Q)} A_{\mu}(n-P-Q+1)\right]\right] \ldots\right]\right]}{\left(k_{1}+\cdots+k_{n-P-Q-1}\right) \cdots k_{n-P-Q-1} k_{n-P-Q}} \\
& =-\sum_{P=1}^{n-2} \sum_{Q=1}^{n-P-1}(-1)^{n-P-Q+1} \frac{\left[A(1),\left[\ldots,\left[A(n-P-Q-1),\left[\widetilde{\delta}_{\Lambda_{1}}^{(P)} A(n-P-Q), \widetilde{\delta}_{\Lambda_{2}}^{(Q)} A_{\mu}(n-P-Q+1)\right]\right] \ldots\right]\right]}{\left(k_{1}+\cdots+k_{n-P-Q-1}\right) \cdots k_{n-P-Q-1} k_{n-P-Q}} .
\end{aligned}
$$

Comparing Eq. (66) for $X_{1 A}$ with Eq. (69) for $X_{1 B}$, leads to the conclusion that

$$
X_{1}=X_{1 A}+X_{1 B}=0
$$

which is the result we intended to prove in this subsection.

\section{B. $X_{2}$}

The terms in Eq. (50) with a factor $A_{\mu}(i)$ without some $\delta_{\Lambda}^{(\ell)}$ acting on them are

$$
\begin{aligned}
X_{2}= & \sum_{\ell=2}^{n-2} \sum_{a=2}^{n-\ell} \frac{(-1)^{a}\left[\overline{\overline{\delta_{\Lambda_{1}}^{(\ell)} A_{\mu}(1)}},\left[A(2),\left[\ldots,\left[A(a-1), \widetilde{\delta}_{\Lambda_{2}}^{(n-\ell-a+1)} A(a)\right] \ldots\right]\right]\right]}{\left(k_{2}+\cdots+k_{a-1}\right) \cdots k_{a-1} k_{a}} \\
& -\sum_{\ell=1}^{n-3} \sum_{a=3}^{n-\ell} \sum_{b=2}^{a-1} \frac{(-1)^{a}}{\left(k_{2}+\cdots+k_{a-1}\right) \cdots k_{a-1} k_{a}}\left[A_{\mu}(1),\left[\ldots,\left[\widetilde{\delta}_{\Lambda_{1}}^{\ell)} A(b),\left[\ldots,\left[A(a-1), \widetilde{\delta}_{\Lambda_{2}}^{(n-\ell-a+1)} A(a)\right] \ldots\right]\right] \ldots\right]\right] \\
& +\sum_{\ell=2}^{n-3} \sum_{a=3}^{n-\ell} \sum_{b=2}^{a-1} \frac{(-1)^{a}}{\left(k_{2}+\cdots+k_{a-1}\right) \cdots k_{a-1} k_{a}}\left[A_{\mu}(1),\left[\ldots,\left[\overline{\delta_{\Lambda_{1}}^{(\ell)} A(b)},\left[\ldots,\left[A(a-1), \widetilde{\delta}_{\Lambda_{2}}^{(n-\ell-a+1)} A(a)\right] \ldots\right]\right] \ldots\right]\right] \\
& +\sum_{\ell=1}^{n-3} \sum_{a=3}^{n-\ell} \frac{(-1)^{a}}{2\left(k_{2}+\cdots+k_{a-2}\right)^{\prime} \cdots k_{a-2} k_{a-1} k_{a}}\left[A_{\mu}(1),\left[A(2),\left[\ldots,\left[A(a-2),\left[\widetilde{\delta}_{\Lambda_{1}}^{(\ell)} A(a-1), \widetilde{\delta}_{\Lambda_{2}}^{(n-\ell-a+1)} A(a)\right]\right] \ldots\right]\right]\right]
\end{aligned}
$$

where, this time, $\overline{\overline{\delta_{\Lambda_{1}}^{(\ell)} A_{\mu}(k)}}$ denotes the term proportional to $A_{\mu}$ in its expansion given by Eq. (34), i.e.,

$$
\overline{\overline{\delta_{\Lambda_{1}}^{(\ell)} A_{\mu}(k)}}=\sum_{c=1}^{\ell-1} \frac{(-1)^{c}\left[A_{\mu}(1),\left[A(2),\left[\cdots,\left[A(c), \widetilde{\delta}_{\Lambda_{1}}^{\ell-c)} A(c+1)\right] \cdots\right]\right]\right.}{\left(k_{2}+\cdots+k_{c}\right) \cdots k_{c} k_{c+1}} .
$$

We want to show that $X_{2}$ vanishes. 
Let us respectively denote by $X_{2 A}, X_{2 B}, X_{2 C}$, and $X_{2 D}$ the four terms in the order in which they appear in Eq. (71) for $X_{2}$. Substituting Eq. (72) into the first term $X_{2 A}$ of Eq. (71) yields

$$
\begin{aligned}
X_{2 A}= & \sum_{\ell=2}^{n-2} \sum_{a=2}^{n-\ell} \sum_{c=1}^{\ell-1}(-1)^{a+c} \frac{\left[\left[A_{\mu}(1),\left[A(2),\left[\ldots,\left[\left[A(c), \widetilde{\delta}_{\Lambda_{1}}^{(\ell-c)} A(c+1)\right] \ldots\right]\right]\right]\right.\right.}{\left(k_{2}+\cdots+k_{c}\right) \cdots k_{c} k_{c+1}} \\
& \frac{\left.\left[A(c+2),\left[\ldots,\left[A(c+a-1), \widetilde{\delta}_{\Lambda_{2}}^{(n-\ell-a+1)} A(c+a)\right] \ldots\right]\right]\right]}{\left(k_{c+2}+\cdots+k_{c+a-1}\right) \cdots k_{c+a-1} k_{c+a}} .
\end{aligned}
$$

If we define $Y_{\Lambda}^{\ell, c}(k)$ by

$$
Y_{\Lambda}^{\ell, c}(k)=\frac{\left[A(1),\left[\ldots,\left[A(c-1), \widetilde{\delta}_{\Lambda}^{(\ell-c)} A(c)\right] \ldots\right]\right]}{\left(k_{1}+\cdots+k_{c-1}\right) \cdots k_{c-1} k_{c}},
$$

then

$$
Y_{\Lambda}^{n-\ell, a-1}(k)=\frac{\left[A(1),\left[\ldots,\left[A(a-2), \widetilde{\delta}_{\Lambda}^{(n-\ell-a+1)} A(a-1)\right] \ldots\right]\right]}{\left(k_{1}+\cdots+k_{a-2}\right) \cdots k_{a-2} k_{a-1}},
$$

and

$$
X_{2 A}=\sum_{\ell=2}^{n-2} \sum_{a=2}^{n-\ell} \sum_{c=1}^{\ell-1}(-1)^{a+c}\left[\left[A_{\mu}(1), Y_{\Lambda_{1}^{\ell, c}}^{\ell}(2)\right], Y_{\Lambda_{2}}^{n-\ell, a-1}(3)\right]
$$

Making the change of variables

$$
L=n-\ell, \quad C=a-1, \quad A=c+1,
$$

in Eq. (76), we see that $X_{2 A}$ can also be written in the form

$$
X_{2 A}=\sum_{L=2}^{n-2} \sum_{C=1}^{L-1} \sum_{A=2}^{n-L}(-1)^{A+C}\left[\left[A_{\mu}(1), Y_{\Lambda_{1}}^{n-L, A-1}(2)\right], Y_{\Lambda_{2}}^{L, C}(3)\right]
$$

Taking half the sum of the expressions (76) and (78) yields

$$
\begin{aligned}
X_{2 A} & =\frac{1}{2} \sum_{\ell=2}^{n-2} \sum_{a=2}^{n-\ell} \sum_{c=1}^{\ell-1}(-1)^{a+c}\left\{\left[\left[A_{\mu}(1), Y_{\Lambda_{1}^{\ell}, c}(2)\right], Y_{\Lambda_{2}}^{n-\ell, a-1}(3)\right]+\left[\left[A_{\mu}(1), Y_{\Lambda_{1}}^{n-\ell, a-1}(2)\right], Y_{\Lambda_{2}}^{\ell, c}(3)\right]\right\} \\
& =\frac{1}{2} \sum_{\ell=2}^{n-2} \sum_{a=2}^{n-\ell} \sum_{c=1}^{\ell-1}(-1)^{a+c}\left[A_{\mu}(1),\left[Y_{\Lambda_{1}, c}^{\ell}(2), Y_{\Lambda_{2}}^{n-\ell, a-1}(3)\right]\right] .
\end{aligned}
$$

In Eq. (79), we made use of the Jacobi identity to write the sum of two commutators as a single term. Substituting the definition (74) for the expressions $Y$ into Eq. (79), then leads to

$$
\begin{aligned}
X_{2 A}= & \frac{1}{2} \sum_{\ell=2}^{n-2} \sum_{a=2}^{n-\ell} \sum_{c=1}^{\ell-1}(-1)^{a+c} \frac{\left[A_{\mu}(1),\left[\left[\bar{A}(1),\left[\ldots,\left[\bar{A}(c-1), \widetilde{\delta}_{\Lambda_{1}}^{\ell-c)} \bar{A}(c)\right] \ldots\right]\right],\right.\right.}{\left(K_{1}+\cdots+K_{c-1}\right) \cdots K_{c-1} K_{c}} \\
& \frac{\left.\left.\left[\bar{A}(c+1),\left[\ldots,\left[\bar{A}(c+a-2), \widetilde{\delta}_{\Lambda_{2}}^{(n-\ell-a+1)} \bar{A}(c+a-1)\right] \ldots\right]\right]\right]\right]}{\left(K_{c+1}+\cdots+K_{c+a-2}\right) \cdots K_{c+a-2} K_{c+a-1}} \\
= & -\frac{1}{2} \sum_{\ell=1}^{n-3} \sum_{a=2}^{n-\ell} \sum_{c=0}^{\ell-1}(-1)^{a+c} \frac{\left[A_{\mu}(1),\left[\left[\bar{A}(1),\left[\ldots,\left[\bar{A}(c), \widetilde{\delta}_{\Lambda_{1}}^{\ell-c)} \bar{A}(c+1)\right] \ldots\right]\right],\right.\right.}{\left(K_{1}+\cdots+K_{c}\right) \cdots K_{c} K_{c+1}} \\
& \frac{\left.\left.\left[\bar{A}(c+2),\left[\ldots,\left[\bar{A}(c+a-1), \widetilde{\delta}_{\Lambda_{2}}^{(n-\ell-a)} \bar{A}(c+a)\right] \ldots\right]\right]\right]\right]}{\left(K_{c+2}+\cdots+K_{c+a-1}\right) \cdots K_{c+a-1} K_{c+a}} .
\end{aligned}
$$

Upon comparing this expression (80) with the formula (67) for $X_{1 B}$, one sees that 


$$
X_{2 A}=\frac{1}{2}\left[A_{\mu}(1), X_{1 B}\left(n \rightarrow n-1, \widetilde{\delta}_{\Lambda} A_{\mu}(c+1) \rightarrow \widetilde{\delta}_{\Lambda} A(c+1) / k_{c+1}\right)\right]
$$

We can then make use of the evaluation of $X_{1 B}$ in Eq. (69) to write

$$
\begin{aligned}
X_{2 A}= & -\frac{1}{2} \sum_{P=1}^{n-3} \sum_{Q=1}^{n-P-2}(-1)^{n-P-Q}\left[A_{\mu}(1),[A(2),[\ldots,\right. \\
& \frac{\left.\left.\left.\left[A(n-P-Q-1),\left[\widetilde{\delta}_{\Lambda_{1}}^{(P)} A(n-P-Q), \widetilde{\delta}_{\Lambda_{2}}^{Q)} A(n-P-Q+1)\right]\right] \ldots\right]\right]\right]}{\left(k_{2}+\cdots+k_{n-P-Q-1}\right) \cdots k_{n-P-Q-1} k_{n-P-Q^{-}} k_{n-P-Q+1}} .
\end{aligned}
$$

We now examine the next two terms, $X_{2 B}$ and $X_{2 C}$, in Eq. (71). They can be combined into one expression in analogy with the manipulations for $X_{1 A}$ :

$$
\begin{aligned}
X_{2 B}+X_{2 C}= & -\sum_{\ell=1}^{n-3} \sum_{a=3}^{n-\ell} \sum_{b=2}^{a-1} \sum_{c=0}^{\ell-1} \frac{(-1)^{a+c}\left[A_{\mu}(1),\left[\ldots,\left[\left[A(b),\left[\ldots,\left[A(b+c-1), \widetilde{\delta}_{\Lambda_{1}}^{\ell-c)} A(b+c)\right] \cdots\right]\right],\right.\right.\right.}{\left(k_{2}+\cdots+k_{a+c-1}\right) \cdots\left(k_{b}+\cdots+k_{a+c-1}\right)\left(k_{b}+\cdots+k_{b+c-1}\right) \cdots k_{b+c-1}} \\
& \frac{\left.\left.\left.\left[A(b+c+1),\left[\ldots,\left[A(a+c-1), \widetilde{\delta}_{\Lambda_{2}}^{(n-\ell-a+1)} A(a+c)\right] \ldots\right]\right]\right] \ldots\right]\right]}{\left(k_{b+c+1}+\cdots+k_{a+c-1}\right) \cdots k_{a+c-1} k_{a+c}} .
\end{aligned}
$$

Here, too, we could extend the summation over $\ell$ to include the term with $\ell=1$. Let $n=n^{\prime}+1$, and shift the summations over $a$ and $b$ by one unit, then

$$
\begin{aligned}
X_{2 B}+X_{2 C}= & \sum_{\ell=1}^{n^{\prime}-2} \sum_{a=2} \sum_{b=1}^{n^{\prime}-\ell} \sum_{c=0}^{a-1} \frac{(-1)^{a+c}\left[A_{\mu}(1),\left[\ldots,\left[\left[A(b+1),\left[\ldots,\left[A(b+c), \widetilde{\delta}_{\Lambda_{1}}^{(\ell-c)} A(b+c+1)\right] \ldots\right]\right],\right.\right.\right.}{\left(k_{2}+\cdots+k_{a+c}\right) \cdots\left(k_{b}+\cdots+k_{a+c}\right)\left(k_{b+1}+\cdots+k_{b+c}\right) \cdots k_{b+c}} \\
& \frac{\left.\left.\left.\left[A(b+c+2),\left[\ldots,\left[A(a+c), \widetilde{\delta}_{\Lambda_{2}}^{\left(n^{\prime}-\ell-a+1\right)} A(a+c+1)\right] \ldots\right]\right]\right] \ldots\right]\right]}{\left(k_{b+c+2}+\cdots+k_{a+c}\right) \cdots k_{a+c} k_{a+c+1}} .
\end{aligned}
$$

One recognizes that this expression is the commutator of $A_{\mu}(1)$ with $X_{1 A}$ with the replacements $n \rightarrow n-1$ and $\widetilde{\delta}_{\Lambda} A_{\mu}(i) \rightarrow \widetilde{\delta}_{\Lambda} A(i) / k_{i}$ [see Eq. (57)]. Hence, we can immediately derive from Eq. (66) that

$$
\begin{aligned}
X_{2 B}+X_{2 C}= & \sum_{P=1} \sum_{Q=1}^{n^{\prime}-2}(-1)^{n^{\prime}-P-Q+1}\left[A_{\mu}(1),[A(2),[\ldots,\right. \\
& \frac{\left.\left.\left.\left[A\left(n^{\prime}-P-Q\right),\left[\widetilde{\delta}_{\Lambda_{1}}^{(P)} A\left(n^{\prime}-P-Q+1\right), \widetilde{\delta}_{\Lambda_{2}}^{(Q)} A\left(n^{\prime}-P-Q+2\right)\right]\right] \ldots\right]\right]\right]}{\left(k_{2}+\cdots+k_{n^{\prime}-P-Q}\right) \cdots k_{n^{\prime}-P-Q^{\prime}} k_{n^{\prime}-P-Q+1} k_{n^{\prime}-P-Q+2}} \\
= & \sum_{P=1}^{n-3} \sum_{Q=1}^{n-P-2}(-1)^{n-P-Q} \\
& \frac{\left[A_{\mu}(1),\left[A(2),\left[\cdots,\left[A(n-P-Q-1),\left[\widetilde{\delta}_{\Lambda_{1}}^{(P)} A(n-P-Q), \widetilde{\delta}_{\Lambda_{2}}^{Q)} A(n-P-Q+1)\right]\right] \ldots\right]\right]\right]}{\left(k_{2}+\cdots+k_{n-P-Q-1}\right) \cdots k_{n-P-Q-1} k_{n-P-Q} k_{n-P-Q+1}} .
\end{aligned}
$$

Finally, we examine the last term $X_{2 D}$ in Eq. (71). A simple change of variables

$$
P=\ell, \quad Q=n-\ell-a+1,
$$

yields

$$
\begin{aligned}
X_{2 D}= & -\sum_{P=1}^{n-3} \sum_{Q=1}^{n-P-2}(-1)^{n-P-Q}\left[A_{\mu}(1),\right. \\
& \frac{\left.\left[A(2),\left[\ldots,\left[A(n-P-Q-1),\left[\widetilde{\delta}_{\Lambda_{1}}^{(P)} A(n-P-Q), \widetilde{\delta}_{\Lambda_{2}}^{(Q)} A(n-P-Q+1)\right]\right] \ldots\right]\right]\right]}{2\left(k_{2}+\cdots+k_{n-P-Q-1}\right) \cdots k_{n-P-Q-1} k_{n-P-Q} k_{n-P-Q+1}} .
\end{aligned}
$$


Adding the results (82) for $X_{2 A}$, Eq. (85) for $X_{2 B}+X_{2 C}$, and Eq. (87) for $X_{2 D}$, now shows that

$$
X_{2}=X_{2 A}+X_{2 B}+X_{2 C}+X_{2 D}=0 .
$$

This is the result we set out to prove in this subsection.

\section{C. $X_{3}$}

Finally, the terms in Eq. (50) with the index $\mu$ in the combination $k_{i \mu}$ are

$$
\begin{aligned}
X_{3}= & \sum_{\ell=1}^{n-2} \sum_{a=2}^{n-\ell} \sum_{b=1}^{a-1} \frac{(-1)^{a}\left(k_{1}+\cdots+k_{a-1}\right)_{\mu}}{\left(k_{1}+\cdots+k_{a-1}\right) \cdots k_{a-1} k_{a}}\left[A(1),\left[\ldots,\left[\widetilde{\delta}_{\Lambda_{1}}^{(\ell)} A(b),\left[\ldots,\left[A(a-1), \widetilde{\delta}_{\Lambda_{2}}^{(n-\ell-a+1)} A(a)\right] \ldots\right]\right] \ldots\right]\right] \\
& -\sum_{\ell=2}^{n-2} \sum_{a=2}^{n-\ell} \sum_{b=1}^{a-1} \frac{(-1)^{a}\left(k_{1}+\cdots+k_{a-1}\right)_{\mu}}{\left(k_{1}+\cdots+k_{a-1}\right) \cdots k_{a-1} k_{a}}\left[A(1),\left[\ldots,\left[\overline{\delta_{\Lambda_{1}}^{(\ell)} A(b)},\left[\ldots,\left[A(a-1), \widetilde{\delta}_{\Lambda_{2}}^{(n-\ell-a+1)} A(a)\right] \ldots\right]\right] \ldots\right]\right] \\
& +\sum_{\ell=2}^{n-2} \sum_{a=2}^{n-\ell} \frac{(-1)^{a}\left[\overline{\overline{\delta_{\Lambda_{1}}^{(\ell)} A_{\mu}(1)}},\left[A(2),\left[\ldots,\left[A(a-1), \widetilde{\delta}_{\Lambda_{2}}^{(n-\ell-a+1)} A(a)\right] \ldots\right]\right]\right]}{\left(k_{2}+\cdots+k_{a-1}\right) \cdots k_{a-1} k_{a}} \\
& -\sum_{\ell=1}^{n-2} \sum_{a=2}^{n-\ell} \frac{(-1)^{a}\left(k_{1}+\cdots+k_{a}\right)_{\mu}}{2\left(k_{1}+\cdots+k_{a-2}\right) \cdots k_{a-2} k_{a-1} k_{a}}\left[A(1),\left[\ldots,\left[A(a-2),\left[\widetilde{\delta}_{\Lambda_{1}}^{(\ell)} A(a-1), \widetilde{\delta}_{\Lambda_{2}}^{(n-\ell-a+1)} A(a)\right]\right] \ldots\right]\right],
\end{aligned}
$$

where, this time, $\overline{\overline{\delta_{\Lambda_{1}}^{(\ell)} A_{\mu}(k)}}$ stands for the second term in its expansion (34), i.e.,

$$
\overline{\overline{\delta_{\Lambda_{1}}^{(\ell)} A_{\mu}(k)}}=-\sum_{c=1}^{\ell-1} \frac{(-1)^{c}\left(k_{1}+\cdots+k_{c}\right)_{\mu}\left[A(1),\left[\ldots,\left[A(c), \widetilde{\delta}_{\Lambda_{1}}^{\ell-c)} A(c+1)\right] \ldots\right]\right]}{\left(k_{1}+\cdots+k_{c}\right) \cdots k_{c} k_{c+1}}
$$

Let us respectively denote by $X_{3 A}, X_{3 B}, X_{3 C}$, and $X_{3 D}$ the four double sums in the order in which they appear in Eq. (89). In analogy with the manipulations for $X_{1 A}$, we can combine $X_{3 A}$ and $X_{3 B}$ into a single expression:

$$
\begin{aligned}
X_{3 A}+X_{3 B}= & \sum_{\ell=1}^{n-2} \sum_{a=2}^{n-\ell} \sum_{b=1}^{a-1} \sum_{c=0}^{\ell-1}(-1)^{a+c}\left(k_{1}+\cdots+k_{c+a-1}\right)_{\mu} \\
& \times \frac{\left[A(1),\left[\ldots,\left[\left[A(b),\left[\ldots,\left[A(c+b-1), \widetilde{\delta}_{\Lambda_{1}}^{\ell-c)} A(c+b)\right] \ldots\right]\right],\right.\right.\right.}{\left(k_{1}+\cdots+k_{c+a-1}\right) \cdots\left(k_{b}+\cdots+k_{c+a-1}\right)} \\
& \frac{\left.\left.\left.\left[A(c+b+1),\left[\ldots,\left[A(c+a-1), \widetilde{\delta}_{\Lambda_{2}}^{(n-\ell-a+1)} A_{\mu}(c+a)\right] \ldots\right]\right]\right] \ldots\right]\right]}{\left(k_{b}+\cdots+k_{c+b-1}\right) \cdots k_{c+b-1}\left(k_{c+b+1}+\cdots+k_{c+a-1}\right) \cdots k_{c+a-1} k_{c+a}} .
\end{aligned}
$$

Comparing the expression (91) with Eq. (57) for $X_{1 A}$, we see that they only differ by the factor $\left(k_{1}+\cdots+k_{c+a-1}\right)_{\mu}$ in the numerator and a factor $k_{c+a}$ in the denominator. Hence, we immediately obtain from Eq. (66) that

$$
\begin{aligned}
X_{3 A}+X_{3 B}= & -\sum_{P=1}^{n-2} \sum_{Q=1}^{n-P-1}(-1)^{n-P-Q}\left(k_{1}+\cdots+k_{n-P-Q}\right)_{\mu}[A(1),[\ldots, \\
& \frac{\left.\left.\left[A(n-P-Q-1),\left[\widetilde{\delta}_{\Lambda_{1}}^{(P)} A(n-P-Q), \widetilde{\delta}_{\Lambda_{2}}^{Q)} A_{\mu}(n-P-Q+1)\right]\right] \cdots\right]\right]}{\left(k_{1}+\cdots+k_{n-P-Q-1}\right) \cdots k_{n-P-Q-1} k_{n-P-Q} k_{n-P-Q+1}} .
\end{aligned}
$$

For the third double sum $X_{3 C}$ in Eq. (89), we can again extend the summation over $\ell$ to include the vanishing term with $\ell=1$ because of (36). But then, we can also include the $c=0$ term, because $\left(k_{1}+\cdots+k_{c}\right)_{\mu}=0$ for $c=0$. Thus, 


$$
\begin{aligned}
X_{3 C}= & -\sum_{\ell=1}^{n-2} \sum_{a=2}^{n-\ell} \sum_{c=0}^{\ell-1}(-1)^{a+c}\left(k_{1}+\cdots+k_{c}\right)_{\mu} \\
& \times \frac{\left[\left[A(1),\left[\ldots,\left[A(c), \widetilde{\delta}_{\Lambda_{1}}^{\ell-c)} A(c+1)\right] \ldots\right]\right],\left[A(c+2),\left[\ldots,\left[A(c+a-1), \widetilde{\delta}_{\Lambda_{2}}^{(n-\ell-a+1)} A(c+a)\right] \ldots\right]\right]\right]}{\left(k_{1}+\cdots+k_{c}\right) \cdots k_{c} k_{c+1}\left(k_{c+2}+\cdots+k_{c+a-1}\right) \cdots k_{c+a-1} k_{c+a}} \\
= & -\sum_{\ell=1}^{n-2} \sum_{a=2}^{n-\ell} \sum_{c=0}^{\ell-1}(-1)^{a+c}\left(k_{a}+\cdots+k_{a+c-1}\right)_{\mu} \\
& \times \frac{\left[\left[A(1),\left[\ldots,\left[A(a-2), \widetilde{\delta}_{\Lambda_{1}}^{(n-\ell-a+1)} A(a-1)\right] \ldots\right]\right],\left[A(a),\left[\ldots,\left[A(a+c-1), \widetilde{\delta}_{\Lambda_{2}}^{(\ell-c)} A(a+c)\right] \ldots\right]\right]\right]}{\left(k_{1}+\cdots+k_{a-2}\right) \cdots k_{a-2} k_{a-1}\left(k_{a}+\cdots+k_{a+c-1}\right) \cdots k_{a+c-1} k_{a+c}},
\end{aligned}
$$

where, in the last step, we merely anticommuted the two terms in the commutator and renamed the integration variables accordingly. Introducing new summation variables defined by

$$
C=a-2, \quad \alpha=c+2, \quad L=n-\ell-1,
$$

we find that $X_{3 C}$ can be written as

$$
\begin{aligned}
X_{3 C}= & -\sum_{L=1}^{n-2} \sum_{\alpha=2}^{n-L} \sum_{C=0}^{L-1}(-1)^{\alpha+C}\left(k_{C+2}+\cdots+k_{C+\alpha-1}\right)_{\mu} \frac{\left[\left[A(1),\left[\ldots,\left[A(C), \widetilde{\delta}_{\Lambda_{1}}^{(L-C)} A(C+1)\right] \ldots\right]\right],\right.}{\left(k_{1}+\cdots+k_{C}\right) \cdots k_{C} k_{C+1}} \\
& \frac{\left.\left[A(C+2),\left[\ldots,\left[A(C+\alpha-1), \widetilde{\delta}_{\Lambda_{2}}^{(n-L-\alpha+1)} A(C+\alpha)\right] \ldots\right]\right]\right]}{\left(k_{C+2}+\cdots+k_{C+\alpha-1}\right) \cdots k_{C+\alpha-1} k_{C+\alpha}} .
\end{aligned}
$$

Hence, $X_{3 C}$ is one half the sum of the two expressions (93) and (95), i.e.,

$$
\begin{aligned}
X_{3 C}= & -\frac{1}{2} \sum_{\ell=1}^{n-2} \sum_{a=2}^{n-\ell} \sum_{c=0}^{\ell-1}(-1)^{a+c}\left(k_{1}+\cdots+k_{c}+k_{c+2}+\cdots+k_{c+a-1}\right)_{\mu} \\
& \times \frac{\left[\left[A(1),\left[\ldots,\left[A(c), \widetilde{\delta}_{\left.\Lambda_{1}-c\right)}^{\ell-c}(c+1)\right] \ldots\right]\right],\left[A(c+2),\left[\ldots,\left[A(c+a-1), \widetilde{\delta}_{\Lambda_{2}}^{(n-\ell-a+1)} A(c+a)\right] \ldots\right]\right]\right]}{\left(k_{1}+\cdots+k_{c}\right) \cdots k_{c} k_{c+1}\left(k_{c+2}+\cdots+k_{c+a-1}\right) \cdots k_{c+a-1} k_{c+a}} .
\end{aligned}
$$

From the comparison of this expression (96) with formula (67) for $X_{1 B}$, we infer from Eq. (69) that

$$
\begin{aligned}
X_{3 C}= & \frac{1}{2} \sum_{P=1}^{n-2} \sum_{Q=1}^{n-P-1}(-1)^{n-P-Q}\left(k_{1}+\cdots+k_{n-P-Q-1}\right)_{\mu}[A(1),[\ldots, \\
& \frac{\left.\left.\left[A(n-P-Q-1),\left[\widetilde{\delta}_{\Lambda_{1}}^{(P)} A(n-P-Q), \widetilde{\delta}_{\Lambda_{2}}^{(Q)} A(n-P-Q+1)\right]\right] \ldots\right]\right]}{\left(k_{1}+\cdots+k_{n-P-Q-1}\right) \cdots k_{n-P-Q-1} k_{n-P-Q} k_{n-P-Q+1}} .
\end{aligned}
$$

For the last term of Eq. (89), denoted by $X_{3 D}$, a change of variables

$$
a=n-P-Q+1, \quad \ell=P, \quad n-\ell-a+1=Q,
$$

suffices to write this term in the form

$$
\begin{aligned}
X_{3 D}= & \sum_{P=1}^{n-2} \sum_{Q=1}^{n-P-1}(-1)^{n-P-Q_{(}}\left(k_{1}+\cdots+k_{n-P-Q+1}\right)_{\mu}[A(1),[\ldots, \\
& \frac{\left.\left.\left[A(n-P-Q-1),\left[\widetilde{\delta}_{\Lambda_{1}}^{(P)} A(n-P-Q), \widetilde{\delta}_{\Lambda_{2}}^{(Q)} A(n-P-Q+1)\right]\right] \ldots\right]\right]}{2\left(k_{1}+\cdots+k_{n-P-Q-1}\right) \cdots k_{n-P-Q-1} k_{n-P-Q} k_{n-P-Q+1}} .
\end{aligned}
$$


The various contributions to $X_{3}$ are thus seen to have the same integrand, except for the $k_{i \mu}$ terms in the numerators. From Eqs. (92), (97), and (99), we learn that the various contributions are proportional to

$$
\begin{gathered}
X_{3 A}+X_{3 B} \rightarrow-\left(k_{1}+\cdots+k_{n-P-Q-1}\right)_{\mu}-\left(k_{n-P-Q}\right)_{\mu}, \\
X_{3 C} \rightarrow \frac{1}{2}\left(k_{1}+\cdots+k_{n-P-Q-1}\right)_{\mu} \\
X_{3 D} \rightarrow \frac{1}{2}\left(k_{1}+\cdots+k_{n-P-Q-1}\right)_{\mu}+\left(k_{n-P-Q}\right)_{\mu} .
\end{gathered}
$$

The different contributions are thus seen to add up to zero, hence, the entire expression $X_{3}=0$.

\section{Group property}

We have established that the three type of terms, $X_{1}, X_{2}$, and $X_{3}$, separately vanish. This implies that

$$
X=X_{1}+X_{2}+X_{3}=0
$$

and thus that the relation (39)

$$
\sum_{\ell=0}^{n-1}\left(\delta_{\Lambda_{2}}^{(\ell)} \widetilde{\delta}_{\Lambda_{1}}^{(n-\ell)}-\widetilde{\delta}_{\widetilde{L}^{(n-\ell)}}^{\varrho)}\right) A_{\mu}(k)=0
$$

is satisfied for all values of $n$.

Note that, from the definition (32) of $\delta_{\Lambda}^{(\ell)} A_{\mu}(k)$, it follows that, for all values of $n$ and $\ell$,

$$
\begin{gathered}
\delta_{\Lambda}^{(\ell)} A_{\mu}(k)=R(k) \widetilde{\delta}_{\Lambda}^{(\varrho)} A_{\mu}(k), \\
\delta_{L^{(n-\ell)}}^{(\varrho)} A_{\mu}(k)=R(k) \widetilde{\delta}_{\widetilde{L}^{(n-\ell)}}^{(\varrho)} A_{\mu}(k) .
\end{gathered}
$$

Hence, multiplying the LHS of Eq. (102) with $R(k)$ proves the group property (38)

$$
\sum_{\ell=0}^{n-1}\left(\delta_{\Lambda_{2}}^{(\ell)} \delta_{\Lambda_{1}}^{(n-\ell)}-\delta_{L^{(n-\ell)}}^{(\ell)}\right) A_{\mu}(k)=0
$$

This is the result we wanted to establish in this section.

\section{DISCUSSIONS}

It remains to comment on the relation of the present work with the earlier approaches. At first sight, this idea of point splitting seems inconsistent with local gauge symmetries, which play such a crucial role in our present understanding of fundamental interactions [4,5]. In order to pinpoint this possible incompatibility, we first examined four years ago the simplest case of an Abelian U(1) symmetry [6]. To our great surprise, there is no inconsistency in this case, a most encouraging result which is responsible for the later developments, including the present paper.

Since the Abelian gauge field $A_{\mu}(x)$ has no selfinteraction, it is necessary to couple it to a charged matter field $\psi(x)$ to generate interactions. For $A_{\mu}$, we used the standard transformation

$$
\delta_{\Lambda} A_{\mu}(x)=-\partial_{\mu} \Lambda(x)
$$

for $\psi(x)$, we made the ansatz

$$
\delta_{\Lambda} \psi(x)=-\imath e \Lambda(x+\epsilon) \psi(x+2 \epsilon)+O\left(e^{2}\right),
$$

and determined the higher order terms in Eq. (106) by requiring that the Abelian character of the gauge transformation be preserved, i.e.,

$$
\left[\delta_{\Lambda_{1}}, \delta_{\Lambda_{2}}\right] \psi(x)=0
$$

What is shown in Ref. [6] is that a solution of Eq. (107) exists to all orders in $e$, meaning that the higher-order terms in Eq. (106) can indeed be derived. This proof consists of showing that, order by order, although the number of conditions far exceeds the number of parameters to be determined, these conditions are such that solutions do exist.

With this unexpected success, it was natural to study next the Yang-Mills SU(2) gauge symmetry [4]. Instead of Eq. (107), we require that, for the gauge fields $\vec{A}_{\mu}$,

$$
\left[\delta_{\Lambda_{1}}, \delta_{\Lambda_{2}}\right] \vec{A}_{\mu}(x)=\delta_{L} \vec{A}_{\mu}(x),
$$

where $\vec{L}$ is the combined gauge parameter. In order to satisfy the requirement of Eq. (108), it was found that it is not possible to use the original form of point splitting of Dirac [1] or some version of Eq. (106). Instead, it is essential to perform an average over the amount of point splitting using a suitable weight function. More precisely, the simplest ansatz consistent with Eq. (108) is [7]

$$
\begin{gathered}
\delta_{\Lambda}^{(0)} \vec{A}_{\mu}(x)=-\partial_{\mu} \vec{\Lambda}(x), \\
\delta_{\Lambda}^{(1)} \vec{A}_{\mu}(x)=\int_{-\infty}^{+\infty} d \xi \rho(\xi) \vec{\Lambda}(x+\xi \epsilon) \times \vec{A}_{\mu}(x-\xi \epsilon) .
\end{gathered}
$$


Up to third order in the gauge coupling constant, the generalized gauge transformation and the combined gauge parameter were explicitly constructed.

This weight function $\rho(\xi)$ has to satisfy three conditions: (1) It has to be a real function of the real variable $\xi$; (2) it must be an even function of $\xi$; (3) it has to satisfy the convolution property

$$
\int_{-\infty}^{+\infty} d \eta \rho(\xi-\eta) \rho(\eta)=\rho(\xi)
$$

It may be noted that the the inverse Fourier transform of $R(k)$ indeed satisfies Eq. (110).

While the treatment of the Abelian case [6] is quite satisfactory, that of the Yang-Mills SU(2) case [7] leaves much to be desired. Specifically, the shortcomings include (a) the infinitesimal generalized gauge transformations are constructed only to order $g^{3}$; and (b) the procedure cannot be easily generalized to other non-Abelian groups. Since neither of these shortcomings are present for the present approach, there is no question that the present one is superior to that of [7].

\section{CONCLUSIONS}

We have shown that, for any Lie algebra, it is possible to construct generalized gauge transformation for which the fields and the gauge parameters are taken at different spacetime points. The separation between the different space-time points is characterized by a fixed four-vector $\epsilon_{\mu}$, and, for the construction of the generalized gauge transformations, we find that it is essential to average over the separation $\epsilon_{\mu}$ with a weight function $\rho(\xi)$. The conditions which the weight function has to satisfy lead, in momentum space, to the introduction of a cutoff for the large momentum components of the fields in the direction of $\epsilon_{\mu}$. We are thus led to consider, in momentum space, generalized gauge transformations which only act on the small momentum components of the fields. Such generalized gauge transformations can be expected to lead to regulating theories, an exciting possibility which still needs to be examined carefully. (38)

We find that it is possible to satisfy the group property

$$
\left[\delta_{\Lambda_{1}}, \delta_{\Lambda_{2}}\right] \mathbf{A}_{\mu}(x)=\delta_{L} \mathbf{A}_{\mu}(x),
$$

for the group elements $\mathbf{A}_{\mu}(x)$, if we take the gauge transformations

$$
\delta_{\Lambda} \mathbf{A}_{\mu}(k)=\sum_{n=0}^{\infty} g^{n} R(\epsilon \cdot k) \widetilde{\delta}_{\Lambda}^{(n)} \mathbf{A}_{\mu}(k),
$$

with

$$
\widetilde{\delta}_{\Lambda}^{(0)} \mathbf{A}_{\mu}(k)={ }_{l} k_{\mu} \boldsymbol{\Lambda}(k)
$$

$$
\begin{aligned}
\widetilde{\delta}_{\Lambda}^{(1)} \mathbf{A}_{\mu}(k)= & -{ }_{\imath} \int d^{4} k_{1} d^{4} k_{2} \delta^{(4)}\left(k-k_{1}-k_{2}\right) \\
& \times R\left(\epsilon \cdot k_{1}\right) R\left(\epsilon \cdot k_{2}\right)\left[\mathbf{\Lambda}\left(k_{1}\right), \mathbf{A}_{\mu}\left(k_{2}\right)\right],
\end{aligned}
$$

and, for $n \geqslant 2$,

$$
\begin{aligned}
\widetilde{\delta}_{\Lambda}^{(n)} \mathbf{A}_{\mu}(k)= & -\sum_{a=1}^{n-1}(-1)^{a} \int d^{4} k_{1} \cdots d^{4} k_{a+1} \delta^{(4)}\left(k-k_{1}-\cdots-k_{a+1}\right) R\left(\boldsymbol{\epsilon} \cdot k_{1}\right) \cdots R\left(\boldsymbol{\epsilon} \cdot k_{a}\right) S\left(\boldsymbol{\epsilon} \cdot k_{a+1}\right) \\
& \times\left\{\frac{\left[\boldsymbol{\epsilon} \cdot \mathbf{A}\left(k_{1}\right),\left[\ldots,\left[\boldsymbol{\epsilon} \cdot \mathbf{A}\left(k_{a}\right), \widetilde{\delta}_{\Lambda}^{(n-a)} \mathbf{A}_{\mu}\left(k_{a+1}\right)\right] \ldots\right]\right]}{\boldsymbol{\epsilon} \cdot\left(k_{1}+\cdots+k_{a}\right) \boldsymbol{\epsilon} \cdot\left(k_{2}+\cdots+k_{a}\right) \cdots \boldsymbol{\epsilon} \cdot k_{a}}\right. \\
& +\frac{\left(k_{1}+\cdots+k_{a}\right)\left[\boldsymbol{\epsilon} \cdot \mathbf{A}\left(k_{1}\right),\left[\ldots,\left[\boldsymbol{\epsilon} \cdot \mathbf{A}\left(k_{a}\right), \boldsymbol{\epsilon} \cdot \widetilde{\delta}_{\Lambda}^{(n-a)} \mathbf{A}\left(k_{a+1}\right)\right] \ldots\right]\right]}{\boldsymbol{\epsilon} \cdot\left(k_{1}+\cdots+k_{a}\right) \boldsymbol{\epsilon} \cdot\left(k_{2}+\cdots+k_{a}\right) \cdots \boldsymbol{\epsilon} \cdot k_{a} \boldsymbol{\epsilon} \cdot k_{a+1}} \\
& \left.-\frac{\left[\mathbf{A}_{\mu}\left(k_{1}\right),\left[\boldsymbol{\epsilon} \cdot \mathbf{A}\left(k_{2}\right),\left[\ldots,\left[\boldsymbol{\epsilon} \cdot \mathbf{A}\left(k_{a}\right), \boldsymbol{\epsilon} \cdot \widetilde{\delta}_{\Lambda}^{(n-a)} \mathbf{A}\left(k_{a+1}\right)\right] \ldots\right]\right]\right.}{\boldsymbol{\epsilon} \cdot\left(k_{2}+\cdots+k_{a}\right) \boldsymbol{\epsilon} \cdot\left(k_{3}+\cdots+k_{a}\right) \cdots \boldsymbol{\epsilon} \cdot k_{a} \boldsymbol{\epsilon} \cdot k_{a+1}}\right\},
\end{aligned}
$$

together with the combined gauge parameter $\mathbf{L}(k)$ given by

$$
\mathbf{L}(k)=\sum_{n=1}^{\infty} g^{n} R(\epsilon \cdot k) \widetilde{\mathbf{L}}^{(n)}(k)
$$

with

$$
\widetilde{\mathbf{L}}^{(1)}(k)=-\imath \int d^{4} k_{1} d^{4} k_{2} \delta^{(4)}\left(k-k_{1}-k_{2}\right) R\left(\epsilon \cdot k_{1}\right) R\left(\epsilon \cdot k_{2}\right)\left[\boldsymbol{\Lambda}_{1}\left(k_{1}\right), \boldsymbol{\Lambda}_{2}\left(k_{2}\right)\right],
$$

and, for $n \geqslant 2$, 


$$
\begin{aligned}
\widetilde{\mathbf{L}}^{(n)}(k)= & -\sum_{a=1}^{n-1}(-1)^{a} \int d^{4} k_{1} \cdots d^{4} k_{a+1} \delta^{(4)}\left(k-k_{1}-\cdots-k_{a+1}\right) R\left(\epsilon \cdot k_{1}\right) \cdots R\left(\epsilon \cdot k_{a}\right) S\left(\boldsymbol{\epsilon} \cdot k_{a+1}\right) \\
& \times \frac{\left[\epsilon \cdot \mathbf{A}\left(k_{1}\right),\left[\ldots,\left[\epsilon \cdot \mathbf{A}\left(k_{a}\right), \widetilde{\mathbf{L}}^{(n-a)}\left(k_{a+1}\right)\right] \ldots\right]\right]}{\epsilon \cdot\left(k_{1}+\cdots+k_{a}\right) \epsilon \cdot\left(k_{2}+\cdots+k_{a}\right) \cdots \epsilon \cdot k_{a}} \\
& -i \sum_{a=1}^{n-2} \sum_{\ell=1}^{n-a-1}(-1)^{a} \int d^{4} k_{1} \cdots d^{4} k_{a+1} \delta^{(4)}\left(k-k_{1}-\cdots-k_{a+1}\right) \\
& \times R(\epsilon \cdot k) R\left(\epsilon \cdot k_{1}\right) \cdots R\left(\epsilon \cdot k_{a-1}\right) S\left(\epsilon \cdot k_{a}\right) S\left(\epsilon \cdot k_{a+1}\right) \\
& \times \frac{\left[\epsilon \cdot \mathbf{A}\left(k_{1}\right),\left[\cdots,\left[\epsilon \cdot \mathbf{A}\left(k_{a-1}\right),\left[\widetilde{\delta}_{\Lambda_{1}}^{\ell} \mathbf{A}\left(k_{a}\right), \widetilde{\delta}_{\Lambda_{2}}^{(n-\ell-a)} \mathbf{A}\left(k_{a+1}\right)\right]\right] \ldots\right]\right]}{\boldsymbol{\epsilon} \cdot\left(k_{1}+\cdots+k_{a-1}\right) \epsilon \cdot\left(k_{2}+\cdots+k_{a-1}\right) \cdots \cdot \epsilon \cdot k_{a-1} \epsilon \cdot k_{a} \epsilon \cdot k_{a+1}} .
\end{aligned}
$$

We also recall the definitions (10) and (15) of the weight functions $R$ and $S$, which appear in Eqs. (112) through (117):

$$
\begin{gathered}
R(\epsilon \cdot k)= \begin{cases}1 & \text { for }|\epsilon \cdot k|<1, \\
0 & \text { for }|\epsilon \cdot k| \geqslant 1,\end{cases} \\
S(\epsilon \cdot k)=1-R(\epsilon \cdot k) .
\end{gathered}
$$

Note that, in this section, we no longer use our simplified notation, which means among other things that the interchange $\boldsymbol{\Lambda}_{1} \leftrightarrow \boldsymbol{\Lambda}_{2}$ should not be performed in Eqs. (116) and (117).

As explained in Sec. III, the formulas (113) and (114) for $\delta_{\Lambda}^{(n)} \mathbf{A}_{\mu}(k)$ as well as Eqs. (116) and (117) for $\mathbf{L}^{(n)}(k)$ are free of singularities in the limit $\epsilon_{\mu} \rightarrow 0$. This is not immediately obvious from the way these formulas are presented. To see this, one needs to express the higher order gauge variations in terms of first order gauge variations, and to examine the singularity structure for the different integration variables separately. This can be done, however, without much difficulty.

Also note that, in the limit $\epsilon_{\mu} \rightarrow 0$, all $\delta_{\Lambda}^{(n)} \mathbf{A}_{\mu}(k)$ and $\mathbf{L}^{(n)} k$, for $n \geqslant 2$, vanish, because $S(0)=0$, and that all $R$ functions tend to unity because $R(0)=1$. It follows that, in this limit, our generalized gauge transformations reduce to the standard ones, i.e., without point splitting.

\section{ACKNOWLEDGMENTS}

The authors gratefully acknowledge several very useful discussions with their colleagues Mark Fannes, Cecilia Jarlskog, Harry Lehmann, Walter Troost, and Antoine Van Proeyen. Special thanks are due to Conrad Newton for his continued interest in our work. T.T.W. thanks the Theory Division of CERN for its kind hospitality. The work of T.T.W. was supported in part by the U.S. Department of Energy under Grant DE-FG02-84ER40158.

\section{APPENDIX A}

One of the ingredients in our procedure to show that the group property can be satisfied, consists of rewriting commutators in terms of nested commutators only. In this appendix, we derive the formula we need for that purpose, i.e.,

$$
\begin{aligned}
& \sum_{\ell=1}^{n-2} \frac{[[A(1),[\ldots,[A(n-\ell-2), B(n-\ell-1)] \ldots]],[A(n-\ell),[\ldots,[A(n-2), C(n-1)] \ldots]]]}{\left(k_{1}+\cdots+k_{n-\ell-2}\right) \cdots k_{n-\ell-2}\left(k_{n-\ell}+\cdots+k_{n-2}\right) \cdots k_{n-2}} \\
& \quad=\frac{[A(1),[\ldots,[A(n-3),[B(n-2), C(n-1)]] \ldots]]}{\left(k_{1}+\cdots+k_{n-3}\right) \cdots k_{n-3}} .
\end{aligned}
$$

In formula (A1), the arguments of the functions $A(m), B(m)$, and $C(m)$ stand for the integration variables $k_{m}$, and the integration over all the variables $k_{m}$ is implicitly assumed. The quantities $B$ and $C$ can be anything, e.g., they can stand for $\widetilde{\delta}_{\Lambda}^{(P)} A_{\mu}(m)$ or $\widetilde{\delta}_{\Lambda}^{(P)} A(m) / k_{m}$.

The proof of Eq. (A1) requires two other identities: one that allows us to rewrite an arbitrary commutator in terms of a sum over nested commutators, and a more elementary relation which allows us to perform this summation. The first required identity reads 


$$
\begin{aligned}
& \frac{[[A(1),[A(2),[\ldots,[A(n), B(n+1)] \ldots]]], C(n+2)]}{\left(k_{1}+\cdots+k_{n}\right) \cdots k_{n}} \\
& \quad \sum_{i=1}^{n+1} \frac{(-1)^{n-i-1}[A(1),[\ldots,[B(i),[\ldots,[A(n+1), C(n+2)] \ldots]] \ldots]]}{\left(k_{1}+\cdots+k_{i-1}\right) \ldots k_{i-1} k_{i+1} \cdots\left(k_{i+1}+\cdots+k_{n+1}\right)} .
\end{aligned}
$$

We use the induction method to prove Eq. (A2). For $n=2$, we have that

$$
\begin{aligned}
\frac{[[A(1),[A(2), B(3)]], C(4)]}{\left(k_{1}+k_{2}\right) k_{2}}= & \frac{[A(1),[[A(2), B(3)], C(4)]}{\left(k_{1}+k_{2}\right) k_{2}}-\frac{[[A(1), B(2)],[A(3), C(4)]]}{\left(k_{1}+k_{3}\right) k_{1}} \\
= & \frac{[A(1),[A(2),[B(3), C(4)]]]}{\left(k_{1}+k_{2}\right) k_{2}}-\frac{[A(1),[B(2),[A(3), C(4)]]]}{\left(k_{1}+k_{3}\right) k_{3}} \\
& -\frac{[A(1),[B(2),[A(3), C(4)]]]}{\left(k_{1}+k_{3}\right) k_{1}}+\frac{[B(1),[A(2),[A(3), C(4)]]]}{\left(k_{2}+k_{3}\right) k_{2}} \\
= & \frac{[A(1),[A(2),[B(3), C(4)]]]}{\left(k_{1}+k_{2}\right) k_{2}}-\frac{[A(1),[B(2),[A(3), C(4)]]]}{k_{1} k_{3}} \\
& +\frac{[B(1),[A(2),[A(3), C(4)]]]}{k_{2}\left(k_{2}+k_{3}\right)},
\end{aligned}
$$

where, in the derivation, we repeatedly used the Jacobi identity. The result (A3) shows that relation (A2) is indeed satisfied for $n=2$.

We now assume that Eq. (A2) can be used for $n \rightarrow n-1$ to prove the desired result of order $n$, i.e.,

$$
\begin{aligned}
\frac{[[A(1),[\ldots,[A(n), B(n+1)] \ldots]], C(n+2)]}{\left(k_{1}+\cdots+k_{n}\right) \cdots k_{n}} & \\
= & \frac{[A(1),[[A(2),[\ldots,[A(n), B(n+1)] \ldots]], C(n+2)]]}{\left(k_{1}+\cdots+k_{n}\right) \cdots k_{n}} \\
& \quad-\frac{[[A(1),[\ldots,[A(n-1), B(n)] \ldots]],[A(n+1), C(n+2)]]}{\left(k_{1}+\cdots+k_{n-1}+k_{n+1}\right)\left(k_{1}+\cdots+k_{n-1}\right) \cdots k_{n-1}} \\
= & \sum_{i=2}^{n+1} \frac{(-1)^{n-i-1}[A(1),[\ldots,[B(i),[\cdots,[A(n+1), C(n+2)] \ldots]] \ldots]]}{\left(k_{1}+\cdots+k_{i-1}+k_{i+1}+\cdots k_{n+1}\right)\left(k_{2}+\cdots+k_{i-1}\right) \cdots k_{i-1} k_{i+1} \cdots\left(k_{i+1}+\cdots+k_{n+1}\right)} \\
& +\sum_{i=1}^{n} \frac{(-1)^{n-i-1}[A(1),[\ldots,[B(i),[\cdots,[A(n+1), C(n+2)] \ldots]] \ldots]]}{\left(k_{1}+\cdots+k_{i-1}+k_{i+1}+\cdots+k_{n+1}\right)\left(k_{1}+\cdots+k_{i-1}\right) \cdots k_{i-1} k_{i+1} \cdots\left(k_{i+1}+\cdots+k_{n}\right)} \\
= & \sum_{i=1}^{n+1} \frac{(-1)^{n-i-1}[A(1),[\cdots,[B(i),[\ldots,[A(n+1), C(n+2)] \ldots]] \ldots]]}{\left(k_{1}+\cdots+k_{i-1}\right) \cdots k_{i-1} k_{i+1} \cdots\left(k_{i+1}+\cdots+k_{n+1}\right)},
\end{aligned}
$$

which proves Eq. (A2). In these manipulations, we used the Jacobi identity in the first step, followed by an application of the lower order formula on the resulting expressions.

The application of formula (A2) to the LHS of Eq. (A1) introduces a double sum, whereas the RHS of that equation is a single term. The formula which is needed to carry out the summations is given by the elementary identity

$$
\sum_{i=0}^{n} \frac{(-1)^{i}}{k_{1} \cdots\left(k_{1}+\cdots+k_{i}\right)\left(k_{i+1}+\cdots+k_{n}\right) \cdots k_{n}}=\delta_{n, 0} .
$$

Because of our summation convention (37), the relation is trivially satisfied for $n=0$ and $n=1$. Clearly, for $n=2$, relation (A5) is also satisfied, because

$$
\frac{1}{\left(k_{1}+k_{2}\right) k_{2}}-\frac{1}{k_{1} k_{2}}+\frac{1}{k_{1}\left(k_{1}+k_{2}\right)}=0 .
$$

Once more, we use the induction method to prove Eq. (A5) for $n \geqslant 3$. Replacing $k_{n} \rightarrow k_{n}+k_{n+1}$ in Eq. (A5) and dividing by $k_{n+1}$ yields, for $n \geqslant 3$, 


$$
\begin{aligned}
0= & \sum_{i=0}^{n-1} \frac{(-1)^{i}}{k_{1} \cdots\left(k_{1}+\cdots+k_{i}\right)\left(k_{i+1}+\cdots+k_{n+1}\right) \cdots k_{n+1}}+\frac{(-1)^{n}}{k_{1} \cdots\left(k_{1}+\cdots+k_{n-1}\right)\left(k_{1}+\cdots+k_{n+1}\right) k_{n+1}} \\
= & \sum_{i=0}^{n+1} \frac{(-1)^{i}}{k_{1} \cdots\left(k_{1}+\cdots+k_{i}\right)\left(k_{i+1}+\cdots+k_{n+1}\right) \cdots k_{n+1}}-\frac{(-1)^{n}}{k_{1} \cdots\left(k_{1}+\cdots+k_{n}\right) k_{n+1}}-\frac{(-1)^{n+1}}{k_{1} \cdots\left(k_{1}+\cdots+k_{n+1}\right)} \\
& +\frac{(-1)^{n}}{k_{1} \cdots\left(k_{1}+\cdots+k_{n-1}\right)\left(k_{1}+\cdots+k_{n+1}\right) k_{n+1}} \\
= & \sum_{i=0}^{n+1} \frac{(-1)^{i}}{k_{1} \cdots\left(k_{1}+\cdots+k_{i}\right)\left(k_{i+1}+\cdots+k_{n+1}\right) \cdots k_{n+1}},
\end{aligned}
$$

which establishes relation (A5) for $n \rightarrow n+1$, hence, by induction, for all values of $n$.

Finally, to prove the identity (A1), we first use the formula (A2) on the LHS of Eq. (A1). This yields

$$
\begin{aligned}
\sum_{\ell=1}^{n-2} & \frac{[[A(1),[\ldots,[A(n-\ell-2), B(n-\ell-1)] \ldots]],[A(n-\ell),[\ldots,[A(n-2), C(n-1)] \ldots]]]}{\left(k_{1}+\cdots+k_{n-\ell-2}\right) \cdots k_{n-\ell-2}\left(k_{n-\ell}+\cdots+k_{n-2}\right) \cdots k_{n-2}} \\
= & \sum_{\ell=1}^{n-2} \sum_{i=1}^{n-\ell-1} \frac{(-1)^{n-\ell-i-1}[A(1),[\ldots,[B(i),[\ldots,[A(n-2), C(n-1)] \ldots]] \ldots]]}{\left(k_{1}+\cdots+k_{i-1}\right) \cdots k_{i-1} k_{i+1} \cdots\left(k_{i+1}+\cdots k_{n-\ell-1}\right)\left(k_{n-\ell}+\cdots+k_{n-2}\right) \cdots k_{n-2}} \\
= & \sum_{i=1}^{n-2} \frac{[A(1),[\ldots,[B(i),[\ldots,[A(n-2), C(n-1)] \ldots]] \ldots]]}{\left(k_{1}+\cdots+k_{i-1}\right) \cdots k_{i-1}} \\
& \times \sum_{\ell=1}^{n-i-1} \frac{(-1)^{n-\ell-i-1}}{k_{i+1} \cdots\left(k_{i+1}+\cdots+k_{n-\ell-1}\right)\left(k_{n-\ell}+\cdots+k_{n-2}\right) \cdots k_{n-2}} .
\end{aligned}
$$

In the last factor, the sum over $\ell$ can be performed using identity (A5). The change of variable $L=n-\ell-i-1$ transforms this sum into

$$
\sum_{L=0}^{n-i-2} \frac{(-1)^{L}}{k_{i+1} \cdots\left(k_{i+1}+\cdots+k_{i+L}\right)\left(k_{i+1+L}+\cdots+k_{n-2}\right) \cdots k_{n-2}}=\delta_{n-i-2,0} .
$$

This $\delta$ symbol tells us that the sum over $i$ in Eq. (A8) is reduced to the term $i=n-2$, which establishes the result (A1).

\section{APPENDIX B}

In this appendix, we prove the following elementary relation:

$$
\sum_{b=1}^{\alpha} \frac{1}{\left(k_{1}+\cdots+k_{\alpha}\right) \cdots\left(k_{b}+\cdots+k_{\alpha}\right)\left(k_{b}+\cdots+k_{\alpha-1}\right) \cdots k_{\alpha-1}}=\frac{1}{\left(k_{1}+\cdots+k_{\alpha-1}\right) \cdots k_{\alpha-1} k_{\alpha}}
$$

which was used in Sec. IV to simplify the result for $X_{1 A}$.

We use again the induction method to prove Eq. (B1). We, therefore, suppose that

$$
\sum_{b=1}^{\alpha-1} \frac{1}{\left(k_{1}+\cdots+k_{\alpha-1}\right) \cdots\left(k_{b}+\cdots+k_{\alpha-1}\right)\left(k_{b}+\cdots+k_{\alpha-2}\right) \cdots k_{\alpha-2}}=\frac{1}{\left(k_{1}+\cdots+k_{\alpha-2}\right) \cdots k_{\alpha-2} k_{\alpha-1}}
$$

is valid. Let us rename the variables $k_{\ell} \rightarrow k_{\ell+1}$ in Eq. (B2), then

$$
\sum_{b=1}^{\alpha-1} \frac{1}{\left(k_{2}+\cdots+k_{\alpha}\right) \cdots\left(k_{b+1}+\cdots+k_{\alpha}\right)\left(k_{b+1}+\cdots+k_{\alpha-1}\right) \cdots k_{\alpha-1}}=\frac{1}{\left(k_{2}+\cdots+k_{\alpha-1}\right) \cdots k_{\alpha-1} k_{\alpha}},
$$

or, with $B=b+1$,

$$
\sum_{B=2}^{\alpha} \frac{1}{\left(k_{2}+\cdots+k_{\alpha}\right) \cdots\left(k_{B}+\cdots+k_{\alpha}\right)\left(k_{B}+\cdots+k_{\alpha-1}\right) \cdots k_{\alpha-1}}=\frac{1}{\left(k_{2}+\cdots+k_{\alpha-1}\right) \cdots k_{\alpha-1} k_{\alpha}} .
$$


Divide Eq. (B4) by $k_{1}+\cdots+k_{\alpha}$, then

$$
\sum_{B=2}^{\alpha} \frac{1}{\left(k_{1}+\cdots+k_{\alpha}\right) \cdots\left(k_{B}+\cdots+k_{\alpha}\right)\left(k_{B}+\cdots+k_{\alpha-1}\right) \cdots k_{\alpha-1}}=\frac{1}{\left(k_{1}+\cdots+k_{\alpha}\right)\left(k_{2}+\cdots+k_{\alpha-1}\right) \cdots k_{\alpha-1} k_{\alpha}} .
$$

Substituting Eq. (B5) into the LHS of Eq. (B1) yields

$$
\begin{aligned}
& \frac{1}{\left(k_{1}+\cdots+k_{\alpha}\right)\left(k_{1}+\cdots+k_{\alpha-1}\right) \cdots k_{\alpha-1}}+\frac{1}{\left(k_{1}+\cdots+k_{\alpha}\right)\left(k_{2}+\cdots+k_{\alpha-1}\right) \cdots k_{\alpha-1} k_{\alpha}} \\
& \quad=\frac{k_{\alpha}+\left(k_{1}+\cdots+k_{\alpha-1}\right)}{\left(k_{1}+\cdots+k_{\alpha}\right)\left(k_{1}+\cdots+k_{\alpha-1}\right) \cdots k_{\alpha-1} k_{\alpha}} \\
& \quad=\frac{1}{\left(k_{1}+\cdots+k_{\alpha-1}\right) \cdots k_{\alpha-1} k_{\alpha}},
\end{aligned}
$$

which is equal to the RHS of Eq. (B1). Because Eq. (B1) is trivially satisfied for $\alpha=1$ and $\alpha=2$, it is thus shown to be valid for all values of $\alpha$.

[1] P. A. M. Dirac, Proc. Cambridge Philos. Soc. 30, 150 (1934).

[2] R. Peierls, Proc. R. Soc. London A146, 420 (1934); J. Schwinger, Phys. Rev. 82, 664 (1951); K. Johnson, in Lectures on Particles and Field Theory, Lectures at Brandeis University 1964, edited by S. Deser and K.W. Ford (Prentice-Hall, Englewood Cliffs, NJ, 1965), Vol. 2, p. 1.

[3] P. Osland and T. T. Wu, Z. Phys. C 55, 569 (1992); 55, 585 (1992); 55, 593 (1992); Phys. Lett. B 291, 315 (1992); C. Newton, P. Osland, and T. T. Wu, Z. Phys. C 61, 441 (1994); C. Newton and T. T. Wu, ibid. 62, 253 (1994).

[4] C. N. Yang and R. L. Mills, Phys. Rev. 96, 191 (1954).
[5] S. L. Glashow, Nucl. Phys. 22, 579 (1961); S. Weinberg, Phys. Rev. Lett. 19, 1264 (1967); A. Salam, in Elementary Particle Theory: Relativistic Groups and Analyticity (Nobel Symposium No. 8), edited by N. Svartholm (Almqvist and Wiksell, Stockholm, 1968), p. 367; S. L. Glashow, J. Iliopoulos, and L. Maiani, Phys. Rev. D 2, 1285 (1970).

[6] R. Gastmans, C. Newton, and T.T. Wu, CERN Report No. CERN-TH.7008/93 (unpublished); Phys. Lett. B 341, 84 (1994).

[7] R. Gastmans, C. Newton, and T. T. Wu, Phys. Rev. D 54, 5302 (1996). 